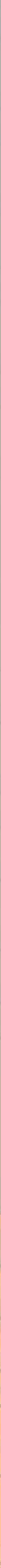




\title{
Network parametrizations for the Grassmannian
}

\author{
Kelli Talaska and Lauren Williams
}

Deodhar introduced his decomposition of partial flag varieties as a tool for understanding Kazhdan-Lusztig polynomials. The Deodhar decomposition of the Grassmannian is also useful in the context of soliton solutions to the KP equation, as shown by Kodama and the second author. Deodhar components $\mathscr{R}_{D}$ of the Grassmannian are in bijection with certain tableaux $D$ called Go-diagrams, and each component is isomorphic to $\left(\mathbb{K}^{*}\right)^{a} \times(\mathbb{K})^{b}$ for some nonnegative integers $a$ and $b$.

Our main result is an explicit parametrization of each Deodhar component in the Grassmannian in terms of networks. More specifically, from a Go-diagram $D$ we construct a weighted network $N_{D}$ and its weight matrix $W_{D}$, whose entries enumerate directed paths in $N_{D}$. By letting the weights in the network vary over $\mathbb{K}$ or $\mathbb{K}^{*}$ as appropriate, one gets a parametrization of the Deodhar component $\Re_{D}$. One application of such a parametrization is that one may immediately determine which Plücker coordinates are vanishing and nonvanishing, by using the Lindström-Gessel-Viennot lemma. We also give a (minimal) characterization of each Deodhar component in terms of Plücker coordinates. A main tool for us is the work of Marsh and Rietsch [Represent. Theory 8 (2004), 212-242] on Deodhar components in the flag variety.

1. Introduction 2276

2. Background on the Grassmannian 2279

3. The main result: network parametrizations from Go-diagrams 2282

4. The Deodhar decomposition of the Grassmannian 2288

5. Formulas for entries of the MR-matrices 2292

6. Proof of the main result 2300

7. A characterization of Deodhar components in terms of Plücker coordinates 2306

Acknowledgements 2310

References $\quad 2310$

The first author was partially supported by NSF grant DMS-1004532. The second author was partially supported by an NSF CAREER award and an Alfred Sloan fellowship.

MSC2010: primary 14M15; secondary 05E10.

Keywords: Grassmannian, network, total positivity, Deodhar decomposition. 


\section{Introduction}

There is a remarkable subset of the real Grassmannian $\mathrm{Gr}_{k, n}(\mathbb{R})$ called its totally nonnegative part $\left(\mathrm{Gr}_{k, n}\right)_{\geq 0}$ [Lusztig 1998; Postnikov 2006], which may be defined as the subset of the real Grassmannian where all Plücker coordinates have the same sign. Postnikov showed that $\left(\mathrm{Gr}_{k, n}\right)_{\geq 0}$ has a decomposition into positroid cells, which are indexed by certain tableaux called J-diagrams. He also gave explicit parametrizations of each cell. In particular, he showed that from each J-diagram one can produce a planar network, and that one can write down a parametrization of the corresponding cell using the weight matrix of that network. This parametrization shows that the cell is isomorphic to $\mathbb{R}_{>0}^{d}$ for some $d$. Such a parametrization is convenient because, for example, one may read off formulas for Plücker coordinates from nonintersecting paths in the network, using the Lindström-Gessel-Viennot lemma.

A natural question is whether these network parametrizations for positroid cells can be extended from $\left(\mathrm{Gr}_{k, n}\right)_{\geq 0}$ to the entire real Grassmannian $\mathrm{Gr}_{k, n}(\mathbb{R})$. In this paper we give an affirmative answer to this question, by replacing the positroid cell decomposition with the Deodhar decomposition of the Grassmannian $\mathrm{Gr}_{k, n}(\mathbb{K})$ (here $\mathbb{K}$ is an arbitrary field).

The components of the Deodhar decomposition are not in general cells, but nevertheless have a simple topology: by [Deodhar 1985; 1987], each one is isomorphic to $\left(\mathbb{K}^{*}\right)^{a} \times(\mathbb{K})^{b}$. The relation of the Deodhar decomposition of $\operatorname{Gr}_{k, n}(\mathbb{R})$ to Postnikov's cell decomposition of $\left(\mathrm{Gr}_{k, n}\right)_{\geq 0}$ is as follows: the intersection of a Deodhar component $\mathscr{R}_{D} \cong\left(\mathbb{R}^{*}\right)^{a} \times(\mathbb{R})^{b}$ with $\left(\mathrm{Gr}_{k, n}\right)_{\geq 0}$ is precisely one positroid cell isomorphic to $\left(\mathbb{R}_{>0}\right)^{a}$ if $b=0$, and is empty otherwise. In particular, when one intersects the Deodhar decomposition with $\left(\mathrm{Gr}_{k, n}\right)_{\geq 0}$, one obtains the positroid cell decomposition of $\left(\mathrm{Gr}_{k, n}\right)_{\geq 0}$. There is a related positroid stratification of the real Grassmannian, and each positroid stratum is a union of Deodhar components.

As for the combinatorics, components of the Deodhar decomposition are indexed by distinguished subexpressions [Deodhar 1985; 1987], or equivalently, by certain tableaux called Go-diagrams [Kodama and Williams 2013], which generalize J-diagrams. In this paper we associate a network to each Go-diagram, and write down a parametrization of the corresponding Deodhar component using the weight matrix of that network. Our construction generalizes Postnikov's, but our networks are no longer planar in general.

Our main results can be summed up as follows. See Theorems 3.16 and 7.8 and the constructions preceding them for complete details.

Theorem. Let $\mathbb{K}$ be an arbitrary field.

- Every point in $\mathrm{Gr}_{k, n}(\mathbb{K})$ can be realized as the weight matrix of a unique network associated to a Go-diagram, and we can explicitly construct the 

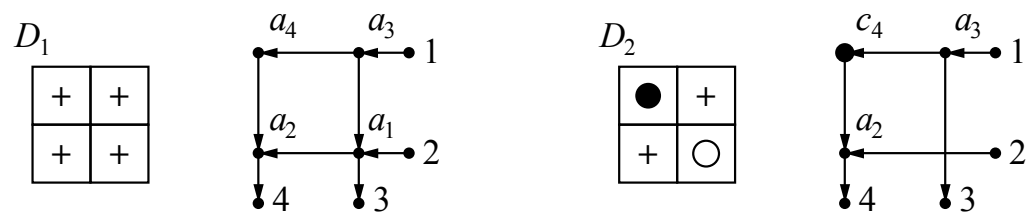

Figure 1. The diagrams and networks associated to $\mathscr{R}_{D_{1}}$ and $\mathscr{R}_{D_{2}}$ in Example 1.1.

corresponding network. The networks corresponding to points in the same Deodhar component have the same underlying graph, but different weights.

- Every Deodhar component may be characterized by the vanishing and nonvanishing of certain Plücker coordinates. Using this characterization, we can also explicitly construct the network associated to a point given either by a matrix representative or by a list of Plücker coordinates.

To illustrate the main results, we provide a small example here. More complicated examples may be seen throughout the rest of the paper.

Example 1.1. Consider the Grassmannian $\mathrm{Gr}_{2,4}$. The large Schubert cell in this Grassmannian can be characterized as

$$
\Omega_{\lambda}=\left\{A \in \mathrm{Gr}_{2,4} \mid \Delta_{1,2}(A) \neq 0\right\},
$$

where $\Delta_{J}$ denotes the Plücker coordinate corresponding to the column set $J$ in a matrix representative of a point in $\mathrm{Gr}_{2,4}$. This Schubert cell contains multiple positroid strata, including $S_{\mathscr{I}}$, where $\mathscr{I}$ is the Grassmann necklace $\mathscr{I}=(12,23,34,14)$. This positroid stratum can also be characterized by the nonvanishing of certain Plücker coordinates:

$$
S_{g}=\left\{A \in \mathrm{Gr}_{2,4} \mid \Delta_{1,2}(A) \neq 0, \Delta_{2,3}(A) \neq 0, \Delta_{3,4}(A) \neq 0, \Delta_{1,4}(A) \neq 0\right\} .
$$

Figure 1 shows two Go-diagrams $D_{1}$ and $D_{2}$ and their associated networks. Note that the network on the right is not planar. The weight matrices associated to these diagrams are

$$
\left(\begin{array}{cccc}
1 & 0 & -a_{3} & -\left(a_{3} a_{4}+a_{3} a_{2}\right) \\
0 & 1 & a_{1} & a_{1} a_{2}
\end{array}\right) \quad \text { and } \quad\left(\begin{array}{cccc}
1 & 0 & -a_{3} & -a_{3} c_{4} \\
0 & 1 & 0 & a_{2}
\end{array}\right) .
$$

The positroid stratum $S_{\mathscr{9}}$ is the disjoint union of the two corresponding Deodhar components $\mathscr{R}_{D_{1}}$ and $\mathscr{R}_{D_{2}}$, which can be characterized in terms of vanishing and nonvanishing of minors as

$$
\mathscr{R}_{D_{1}}=\left\{A \in S_{\mathscr{9}} \mid \Delta_{1,3} \neq 0\right\} \quad \text { and } \quad \mathscr{R}_{D_{2}}=\left\{A \in S_{\mathscr{9}} \mid \Delta_{1,3}=0\right\} .
$$

Note that if one lets the $a_{i}$ 's range over $\mathbb{K}^{*}$ and lets $c_{4}$ range over $\mathbb{K}$, then we see 
that $\mathscr{R}_{D_{1}} \cong\left(\mathbb{K}^{*}\right)^{4}$ and $\mathscr{R}_{D_{2}} \cong\left(\mathbb{K}^{*}\right)^{2} \times \mathbb{K}$.

There are several applications of our construction. First, as a special case of our theorem, one may parametrize all $k \times n$ matrices using networks. Second, by applying the Lindström-Gessel-Viennot lemma to a given network, one may write down explicit formulas for Plücker coordinates in terms of collections of nonintersecting paths in the network. Third, building upon [Kodama and Williams 2013], we obtain (minimal) descriptions of Deodhar components in the Grassmannian in terms of vanishing and nonvanishing of Plücker coordinates. It follows that each Deodhar component is a union of matroid strata.

Although less well known than the Schubert decomposition and matroid stratification, the Deodhar decomposition is very interesting in its own right. Deodhar's original motivation for introducing his decomposition was the desire to understand Kazhdan-Lusztig polynomials. In the flag variety, one may intersect two opposite Schubert cells, obtaining a Richardson variety, which Deodhar showed is a union of Deodhar components. Each Richardson variety $\mathscr{R}_{v, w}(q)$ may be defined over a finite field $\mathbb{K}=\mathbb{F}_{q}$, and in this case the number of points determines the $R$-polynomials $R_{v, w}(q)=\#\left(\mathscr{R}_{v, w}\left(\mathbb{F}_{q}\right)\right)$, introduced by Kazhdan and Lusztig [1979] to give a recursive formula for the Kazhdan-Lusztig polynomials. Since each Deodhar component is isomorphic to $\left(\mathbb{F}_{q}^{*}\right)^{a} \times\left(\mathbb{F}_{q}\right)^{b}$ for some $a$ and $b$, if one understands the decomposition of a Richardson variety into Deodhar components, then in principle one may compute the $R$-polynomials and hence Kazhdan-Lusztig polynomials.

Another reason for our interest in the Deodhar decomposition is its relation to soliton solutions of the KP equation. It is well known that from each point $A$ in the real Grassmannian one may construct a soliton solution $u_{A}(x, y, t)$ of the KP equation. It was shown in [Kodama and Williams 2013] that when the time variable $t$ tends to $-\infty$, the combinatorics of the solution $u_{A}(x, y, t)$ depends precisely on which Deodhar component $A$ lies in.

One final result of this paper is the verification that two notions of total positivity for the Grassmannian coincide. Lusztig [1998] defined the totally nonnegative part of any partial flag variety in a Lie-theoretic way. He also conjectured a cell decomposition for it, proved by Rietsch [1998]. Independently, Postnikov defined the totally nonnegative part of the real Grassmannian in terms of Plücker coordinates, and gave a cell decomposition of it. It is not obvious that Lusztig's definitions (for $\mathrm{Gr}_{k, n}(\mathbb{R})$ ) coincide with Postnikov's; however, this has been verified by Rietsch (personal communication, 2009). In this paper we give a new proof that the two notions of total positivity coincide.

Corollary 1.2. Lusztig's definition of the totally nonnegative part of $\mathrm{Gr}_{k, n}(\mathbb{R})$ and its cell decomposition coincides with Postnikov's definition of the totally nonnegative part of $\mathrm{Gr}_{k, n}(\mathbb{R})$ and its cell decomposition. 
The outline of this paper is as follows. In Section 2 we give some background on the Grassmannian and its decompositions, including the Schubert decomposition, the positroid stratification, and the matroid stratification. In Section 3 we present our main construction: we explain how to construct a network from each diagram, then use that network to write down a parametrization of a subset of the Grassmannian that we call a network component. In Section 4 we define Deodhar's decomposition of the flag variety, and its projection to the Grassmannian. We also describe parametrizations of Deodhar components in the flag variety which are due to Marsh and Rietsch [2004]. In Sections 5 and 6 we prove that after a rational transformation of variables, our network parametrizations coincide with the projections of the Marsh-Rietsch parametrizations. Finally, in Section 7 we give a characterization of Deodhar components in terms of the vanishing and nonvanishing of certain Plücker coordinates.

\section{Background on the Grassmannian}

The Grassmannian $\mathrm{Gr}_{k, n}$ is the space of all $k$-dimensional subspaces of an $n$ dimensional vector space $\mathbb{K}^{n}$. In this paper we will usually let $\mathbb{K}$ be an arbitrary field, though we will often think of it as $\mathbb{R}$ or $\mathbb{C}$. An element of $\mathrm{Gr}_{k, n}$ can be viewed as a full-rank $k \times n$ matrix modulo left multiplication by nonsingular $k \times k$ matrices. In other words, two $k \times n$ matrices represent the same point in $\mathrm{Gr}_{k, n}$ if and only if they can be obtained from each other by row operations. Let $\left(\begin{array}{c}{[n]} \\ k\end{array}\right)$ be the set of all $k$-element subsets of $[n]:=\{1, \ldots, n\}$. For $I \in\left(\begin{array}{c}{[n]} \\ k\end{array}\right)$, let $\Delta_{I}(A)$ be the Plücker coordinate, that is, the maximal minor of the $k \times n$ matrix $A$ located in the column set $I$. The map $A \mapsto\left(\Delta_{I}(A)\right)$, where $I$ ranges over $\left(\begin{array}{c}{[n]} \\ k\end{array}\right)$, induces the Plücker embedding $\mathrm{Gr}_{k, n} \hookrightarrow \mathbb{K} \mathbb{P}\left(\begin{array}{l}n \\ k\end{array}\right)-1$ into projective space.

We now describe several useful decompositions of the Grassmannian: the Schubert decomposition, the positroid stratification, and the matroid stratification. Note that the matroid stratification refines the positroid stratification, which refines the Schubert decomposition. The main subject of this paper is the Deodhar decomposition of the Grassmannian, which refines the positroid stratification, and is refined by the matroid stratification (as we prove in Corollary 7.9).

2A. The Schubert decomposition of $\mathbf{G r}_{k, n}$. Throughout this paper, we identify partitions with their Young diagrams. Recall that the partitions $\lambda$ contained in a $k \times(n-k)$ rectangle are in bijection with $k$-element subset $I \subset[n]$. The boundary of the Young diagram of such a partition $\lambda$ forms a lattice path from the upper-right corner to the lower-left corner of the rectangle. Let us label the $n$ steps in this path by the numbers $1, \ldots, n$, and define $I=I(\lambda)$ as the set of labels on the $k$ vertical steps in the path. Conversely, we let $\lambda(I)$ denote the partition corresponding to the subset $I$. 
Definition 2.1. For each partition $\lambda$ contained in a $k \times(n-k)$ rectangle, we define the Schubert cell

$\Omega_{\lambda}=\left\{A \in \mathrm{Gr}_{k, n} \mid I(\lambda)\right.$ is the lexicographically minimal subset such that $\left.\Delta_{I(\lambda)}(A) \neq 0\right\}$.

As $\lambda$ ranges over the partitions contained in a $k \times(n-k)$ rectangle, this gives the Schubert decomposition of the Grassmannian $\mathrm{Gr}_{k, n}$, that is,

$$
\mathrm{Gr}_{k, n}=\bigsqcup_{\lambda \subset(n-k)^{k}} \Omega_{\lambda}
$$

We now define the shifted linear order $<_{i}$ (for $i \in[n]$ ) to be the total order on $[n]$ defined by

$$
i<_{i} i+1<_{i} i+2<_{i} \cdots<_{i} n<_{i} 1<_{i} \cdots<_{i} i-1 .
$$

One can then define cyclically shifted Schubert cells:

Definition 2.2. For each partition $\lambda$ contained in a $k \times(n-k)$ rectangle, and each $i \in[n]$, we define the cyclically shifted Schubert cell

$\Omega_{\lambda}^{i}=\left\{A \in \mathrm{Gr}_{k, n} \mid I(\lambda)\right.$ is the lexicographically minimal subset with respect to $<_{i}$ such that $\left.\Delta_{I(\lambda)} \neq 0\right\}$.

2B. The positroid stratification of $\mathbf{G r}_{k, n}$. The positroid stratification of the Grassmannian $\mathrm{Gr}_{k, n}$ is obtained by taking the simultaneous refinement of the $n$ Schubert decompositions with respect to the $n$ shifted linear orders $<_{i}$. This stratification was first considered by Postnikov [2006], who showed that the strata are conveniently described in terms of Grassmann necklaces, as well as decorated permutations and I-diagrams. Postnikov coined the terminology positroid because the intersection of the positroid stratification of the real Grassmannian with the totally nonnegative part of the Grassmannian $\left(\mathrm{Gr}_{k, n}\right)_{\geq 0}$ gives a cell decomposition of $\left(\mathrm{Gr}_{k, n}\right)_{\geq 0}$ (whose cells are called positroid cells).

Definition 2.3 [Postnikov 2006, Definition 16.1]. A Grassmann necklace is a sequence $\mathscr{I}=\left(I_{1}, \ldots, I_{n}\right)$ of subsets $I_{r} \subset[n]$ such that, for $i \in[n]$, if $i \in I_{i}$ then $I_{i+1}=\left(I_{i} \backslash\{i\}\right) \cup\{j\}$, for some $j \in[n]$ ( $j$ may coincide with $\left.i\right)$; and if $i \notin I_{i}$ then $I_{i+1}=I_{i}$. (Here indices $i$ are taken modulo $n$.) In particular, we have $\left|I_{1}\right|=\cdots=\left|I_{n}\right|$, which is equal to some $k \in[n]$. We then say that $\mathscr{I}$ is a Grassmann necklace of type $(k, n)$.

Example 2.4. $\mathscr{I}=(1345,3456,3456,4567,4567,1467,1478,1348)$ is an example of a Grassmann necklace of type $(4,8)$. 


\begin{tabular}{|l|l|l|l|}
\cline { 1 - 2 }+ & 0 & + & + \\
\hline+ & 0 & + & \\
\cline { 1 - 2 } 0 & 0 & + & \\
\cline { 1 - 2 }+ & 0 & \multicolumn{2}{|c}{} \\
\cline { 1 - 2 } & &
\end{tabular}

Figure 2. A J-diagram $L=(\lambda, D)_{k, n}$.

Lemma 2.5 [Postnikov 2006, Lemma 16.3]. For $A \in \mathrm{Gr}_{k, n}$, let $\Phi(A)=\left(I_{1}, \ldots, I_{n}\right)$ be the sequence of subsets in $[n]$ such that, for $i \in[n], I_{i}$ is the lexicographically minimal subset of $\left(\begin{array}{c}{[n]} \\ k\end{array}\right)$ with respect to the shifted linear order $<_{i}$ such that $\Delta_{I_{i}}(A) \neq 0$. Then $\Phi(A)$ is a Grassmann necklace of type $(k, n)$.

The positroid stratification of $\mathrm{Gr}_{k, n}$ is defined as follows.

Definition 2.6. Let $\mathscr{I}=\left(I_{1}, \ldots, I_{n}\right)$ be a Grassmann necklace of type $(k, n)$. The positroid stratum $S_{\mathscr{I}}$ is defined to be

$$
S_{\mathscr{I}}=\left\{A \in \mathrm{Gr}_{k, n} \mid \mathscr{I}(A)=\mathscr{I}\right\} .
$$

Equivalently, each positroid stratum is an intersection of $n$ cyclically shifted Schubert cells, that is,

$$
S_{\Phi}=\bigcap_{i=1}^{n} \Omega_{\lambda\left(I_{i}\right)}^{i} .
$$

Grassmann necklaces are in bijection with tableaux called J-diagrams.

Definition 2.7 [Postnikov 2006, Definition 6.1]. Fix $k, n$. A J-diagram $(\lambda, D)_{k, n}$ of type $(k, n)$ is a partition $\lambda$ contained in a $k \times(n-k)$ rectangle together with a filling $D: \lambda \rightarrow\{0,+\}$ of its boxes which has the J-property: there is no 0 which has a + above it and a + to its left. ${ }^{1}$ (Here, "above" means above and in the same column, and "to its left" means to the left and in the same row.)

In Figure 2 we give an example of a J-diagram.

\section{C. The matroid stratification of $\mathbf{G r}_{k, n}$.}

Definition 2.8. A matroid of rank $k$ on the set $[n]$ is a nonempty collection $\mu \subset\left(\begin{array}{c}{[n]} \\ k\end{array}\right)$ of $k$-element subsets in $[n]$, called bases of $\mathcal{M}$, that satisfies the exchange axiom: For any $I, J \in \mathcal{M}$ and $i \in I$ there exists $j \in J$ such that $(I \backslash\{i\}) \cup\{j\} \in \mathcal{M}$.

Given an element $A \in \mathrm{Gr}_{k, n}$, there is an associated matroid $\mu_{A}$ whose bases are the $k$-subsets $I \subset[n]$ such that $\Delta_{I}(A) \neq 0$.

${ }^{1}$ This forbidden pattern is in the shape of a backwards L, and hence is denoted J, pronounced "Le." 
Definition 2.9. Let $M \subset\left(\begin{array}{c}{[n]} \\ k\end{array}\right)$ be a matroid. Define the matroid stratum $S_{\mathcal{M}}$ as

$$
S_{\mathcal{M}}=\left\{A \in \operatorname{Gr}_{k, n} \mid \Delta_{I}(A) \neq 0 \text { if and only if } I \in \mathcal{M}\right\} .
$$

This gives a stratification of $\mathrm{Gr}_{k, n}$ called the matroid stratification, or GelfandSerganova stratification.

Remark 2.10. Clearly the matroid stratification refines the positroid stratification, which in turn refines the Schubert decomposition.

\section{The main result: network parametrizations from Go-diagrams}

In this section we define certain tableaux called Go-diagrams, then explain how to parametrize the Grassmannian using networks associated to Go-diagrams. First we will define more general tableaux called diagrams.

\section{A. Diagrams and networks.}

Definition 3.1. Let $\lambda$ be a partition contained in a $k \times(n-k)$ rectangle. A diagram in $\lambda$ is an arbitrary filling of the boxes of $\lambda$ with pluses + , black stones $\bullet$, and white stones $\bigcirc$.

To each diagram $D$ we associate a network $N_{D}$ as follows.

Definition 3.2. Let $\lambda$ be a partition with $\ell$ boxes contained in a $k \times(n-k)$ rectangle, and let $D$ be a diagram in $\lambda$. Label the boxes of $\lambda$ from 1 to $\ell$, starting from the rightmost box in the bottom row, then reading right to left across the bottom row, then right to left across the row above that, etc. The (weighted) network $N_{D}$ associated to $D$ is a directed graph obtained as follows:

- Associate an internal vertex to each + and each $\bullet$

- After labeling the southeast border of the Young diagram with the numbers $1,2, \ldots, n$ (from northeast to southwest), associate a boundary vertex to each number.

- From each internal vertex, draw an edge right to the nearest +-vertex or boundary vertex.

- From each internal vertex, draw an edge down to the nearest +-vertex or boundary vertex.

- Direct all edges left and down. After doing so, $k$ of the boundary vertices become sources and the remaining $n-k$ boundary vertices become sinks.

- If $e$ is a horizontal edge whose left vertex is a +-vertex (respectively $\bullet$-vertex) in box $b$, assign $e$ the weight $a_{b}$ (respectively $c_{b}$ ). We think of $a_{b}$ and $c_{b}$ as indeterminates, but later they will be elements of $\mathbb{K}^{*}$ and $\mathbb{K}$, respectively.

- If $e$ is a vertical edge, assign $e$ the weight 1 . 


\begin{tabular}{|l|l|l|l|}
\hline+ & + & + & + \\
\cline { 1 - 2 }+ & 0 & + & \\
\cline { 1 - 2 } & + & $\bigcirc$ & \\
\cline { 1 - 2 }+ & $\bigcirc$ & \multicolumn{2}{|c}{} \\
\cline { 1 - 2 } & &
\end{tabular}

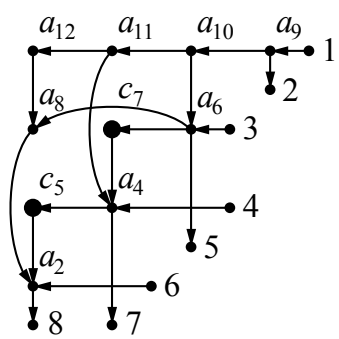

Figure 3. An example of a diagram and its associated network.

Note that in general such a directed graph is not planar, as two edges may cross over each other without meeting at a vertex. See Figure 3 for an example of a diagram and its associated network.

We now explain how to associate a weight matrix to such a network.

Definition 3.3. Let $N_{D}$ be a network as in Definition 3.2. Let

$$
I=\left\{i_{1}<i_{2}<\cdots<i_{k}\right\} \subset[n]
$$

denote the sources. If $P$ is a directed path in the network, let $w(P)$ denote the product of all weights along $P$. If $P$ is the empty path which starts and ends at the same boundary vertex, we let $w(P)=1$. If $s$ is a source and $t$ is any boundary vertex, define

$$
W_{s t}= \pm \sum_{P} w(P),
$$

where the sum is over all paths $P$ from $s$ to $t$. The sign is chosen (uniquely) so that

$$
\Delta_{I \backslash\{s\} \cup\{t\}}\left(W_{D}\right)=\sum_{P} w(P),
$$

where

$$
W_{D}=\left(W_{s t}\right)
$$

is the $k \times n$ weight matrix. We make the convention that the rows of $W_{D}$ are indexed by the sources $i_{1}, \ldots, i_{k}$ from top to bottom, and its columns are indexed by $1,2, \ldots, n$ from left to right. An equivalent way to define the sign of $W_{s t}$ is to let $q=|\{s+1, s+2, \ldots, t-1\} \cap I|$, that is, the number of sources which are strictly between $s$ and $t$. Then the sign of $W_{s t}$ is $(-1)^{q}$.

Example 3.4. The weight matrix associated to the network in Figure 3 is

$$
\left(\begin{array}{cccccccc}
1 & a_{9} & 0 & 0 & a_{9} a_{10} & 0 & -a_{9} a_{10}\left(a_{11}+c_{7}\right) & -a_{9} a_{10}\left(a_{11} a_{12}+a_{11} c_{5}+a_{8}+c_{7} c_{5}\right) \\
0 & 0 & 1 & 0 & -a_{6} & 0 & a_{6} c_{7} & a_{6} a_{8}+a_{6} c_{7} c_{5} \\
0 & 0 & 0 & 1 & 0 & 0 & -a_{4} & -a_{4} c_{5} \\
0 & 0 & 0 & 0 & 0 & 1 & 0 & a_{2}
\end{array}\right)
$$


3B. Distinguished expressions. We now review the notion of distinguished subexpressions, as in [Deodhar 1985] and [Marsh and Rietsch 2004]. This definition will be essential for defining Go-diagrams. We assume the reader is familiar with the (strong) Bruhat order $<$ on $W=\mathfrak{S}_{n}$, and the basics of reduced expressions, as in [Björner and Brenti 2005].

Let $\boldsymbol{w}:=s_{i_{1}} \cdots s_{i_{m}}$ be a reduced expression for $w \in W$. A subexpression $\boldsymbol{v}$ of $\boldsymbol{w}$ is a word obtained from the reduced expression $\boldsymbol{w}$ by replacing some of the factors with 1 . For example, consider a reduced expression in $\mathfrak{S}_{4}$, say $s_{3} s_{2} s_{1} s_{3} s_{2} s_{3}$. Then $s_{3} s_{2} 1 s_{3} s_{2} 1$ is a subexpression of $s_{3} s_{2} s_{1} s_{3} s_{2} s_{3}$. Given a subexpression $\boldsymbol{v}$, we set $v_{(k)}$ to be the product of the leftmost $k$ factors of $\boldsymbol{v}$, if $k \geq 1$, and $v_{(0)}=1$.

Definition 3.5 [Marsh and Rietsch 2004; Deodhar 1985]. Given a subexpression $\boldsymbol{v}$ of a reduced expression $\boldsymbol{w}=s_{i_{1}} s_{i_{2}} \cdots s_{i_{m}}$, we define

$$
\begin{aligned}
J_{v}^{\circ} & :=\left\{k \in\{1, \ldots, m\} \mid v_{(k-1)}<v_{(k)}\right\}, \\
J_{v}^{+} & :=\left\{k \in\{1, \ldots, m\} \mid v_{(k-1)}=v_{(k)}\right\}, \\
J_{\boldsymbol{v}}^{\bullet} & :=\left\{k \in\{1, \ldots, m\} \mid v_{(k-1)}>v_{(k)}\right\} .
\end{aligned}
$$

The expression $\boldsymbol{v}$ is called nondecreasing if $v_{(j-1)} \leq v_{(j)}$ for all $j=1, \ldots, m$, for example, if $J_{\boldsymbol{v}}^{\bullet}=\varnothing$.

Definition 3.6 (distinguished subexpressions [Deodhar 1985, Definition 2.3]). A subexpression $\boldsymbol{v}$ of $\boldsymbol{w}$ is called distinguished if we have

$$
v_{(j)} \leq v_{(j-1)} s_{i_{j}} \text { for all } j \in\{1, \ldots, m\} .
$$

In other words, if right multiplication by $s_{i_{j}}$ decreases the length of $v_{(j-1)}$, then in a distinguished subexpression we must have $v_{(j)}=v_{(j-1)} s_{i_{j}}$.

We write $\boldsymbol{v} \prec \boldsymbol{w}$ if $\boldsymbol{v}$ is a distinguished subexpression of $\boldsymbol{w}$.

Definition 3.7 (positive distinguished subexpressions). We call a subexpression $v$ of $\boldsymbol{w}$ a positive distinguished subexpression (or a PDS for short) if

$$
v_{(j-1)}<v_{(j-1)} s_{i_{j}} \text { for all } j \in\{1, \ldots, m\} .
$$

In other words, it is distinguished and nondecreasing.

Lemma 3.8 [Marsh and Rietsch 2004]. Given $v \leq w$ and a reduced expression $\boldsymbol{w}$ for $w$, there is a unique PDS $\boldsymbol{v}_{+}$for $v$ in $\boldsymbol{w}$.

3C. Go-diagrams. In this section we explain how to index distinguished subexpressions by certain tableaux called Go-diagrams, which were introduced in [Kodama and Williams 2013]. Go-diagrams are certain fillings of Young diagrams by pluses + , black stones $\bullet$, and white stones $\bigcirc .^{2}$

${ }^{2}$ In [Kodama and Williams 2013], we used a slightly different convention and used blank boxes in place of +'s. 


\begin{tabular}{|l|l|l|l|l|}
\hline$S_{5}$ & $S_{4}$ & $S_{3}$ & $S_{2}$ & $S_{1}$ \\
\hline$S_{6}$ & $S_{5}$ & $S_{4}$ & $S_{3}$ & $S_{2}$ \\
\hline$S_{7}$ & $S_{6}$ & $S_{5}$ & $S_{4}$ & $S_{3}$ \\
\hline
\end{tabular}

\begin{tabular}{|c|c|c|c|c|}
\hline 15 & 14 & 13 & 12 & 11 \\
\hline 10 & 9 & 8 & 7 & 6 \\
\hline 5 & 4 & 3 & 2 & 1 \\
\hline
\end{tabular}

\begin{tabular}{|l|l|l|l|l|}
\hline 15 & 12 & 9 & 6 & 3 \\
\hline 14 & 11 & 8 & 5 & 2 \\
\hline 13 & 10 & 7 & 4 & 1 \\
\hline
\end{tabular}

Figure 4. The labeling of a the boxes of a partition by simple generators $s_{i}$, and two reading orders.

Fix $k$ and $n$. Let $W_{k}=\left\langle s_{1}, s_{2}, \ldots, \hat{s}_{n-k}, \ldots, s_{n-1}\right\rangle$ be a parabolic subgroup of $W=\mathfrak{S}_{n}$. Let $W^{k}$ denote the set of minimal-length coset representatives of $W / W_{k}$. Recall that a descent of a permutation $\pi$ is a position $j$ such that $\pi(j)>\pi(j+1)$. Then $W^{k}$ is the subset of permutations of $\mathfrak{S}_{n}$ which have at most one descent; and that descent must be in position $n-k$.

It is well known that elements $w$ of $W^{k}$ can be identified with partitions $\lambda_{w}$ contained in a $k \times(n-k)$ rectangle: if $w=\left(w_{1}, \ldots, w_{n}\right) \in W^{k}$ then

$$
\left\{w_{n-k+1}, w_{n-k+2}, \ldots, w_{n}\right\}
$$

is a subset of size $k$, which gives rise to a partition, as described at the beginning of Section 2A. We refer to this partition as $\lambda_{w}$.

Moreover, it follows from [Stembridge 1996] and [Proctor 1984] that the reduced expressions of $w \in W^{k}$ correspond to certain reading orders of the boxes of the partition $\lambda_{w}$. Specifically, let $Q^{k}$ be the poset whose elements are the boxes of a $k \times(n-k)$ rectangle; if $b_{1}$ and $b_{2}$ are two adjacent boxes such that $b_{2}$ is immediately to the left or immediately above $b_{1}$, we have a cover relation $b_{1} \lessdot b_{2}$ in $Q^{k}$. The partial order on $Q^{k}$ is the transitive closure of $\lessdot$. Now label the boxes of the rectangle with simple generators $s_{i}$ as in Figure 4. If $b$ is a box of the rectangle, then let $s_{b}$ denote its label by a simple generator. Let $w_{0}^{k} \in W^{k}$ denote the longest element in $W^{k}$. Then the set of reduced expressions of $w_{0}^{k}$ can be obtained by choosing a linear extension of $Q^{k}$ and writing down the corresponding word in the $s_{i}$ 's. We call such a linear extension a reading order; two linear extensions are shown in Figure 4. Additionally, given a partition $\lambda_{w}$ contained in the $k \times(n-k)$ rectangle (chosen so that the upper-left corner of its Young diagram is aligned with the upper-left corner of the rectangle), and a linear extension of the subposet of $Q^{k}$ comprised of the boxes of $\lambda$, the corresponding word in $s_{i}$ 's is a reduced expression of the permutation $w \in W^{k}$. Moreover, all reduced expressions of $w$ can be obtained by varying the linear extension.

Definition 3.9 [Kodama and Williams 2013, Section 4]. Fix $k$ and $n$. Let $w \in W^{k}$, let $\boldsymbol{w}$ be a reduced expression for $w$, and let $\boldsymbol{v}$ be a distinguished subexpression of $\boldsymbol{w}$. Then $w$ and $\boldsymbol{w}$ determine a partition $\lambda_{w}$ contained in a $k \times(n-k)$ rectangle together with a reading order of its boxes. The Go-diagram associated to $\boldsymbol{v}$ and $\boldsymbol{w}$ 
is a filling of $\lambda_{w}$ with pluses and black and white stones, such that: for each $k \in J_{v}^{\circ}$ we place a white stone in the corresponding box; for each $k \in J_{v}^{\bullet}$ we place a black stone in the corresponding box of $\lambda_{w}$; and for each $k \in J_{v}^{+}$we place a plus in the corresponding box of $\lambda_{w}$.

Remark 3.10. By [Kodama and Williams 2013, Section 4], whether or not a filling of a partition $\lambda_{w}$ is a Go-diagram does not depend on the choice of reading order of the boxes of $\lambda_{w}$.

Definition 3.11. We define the standard reading order of the boxes of a partition to be the reading order which starts at the rightmost box in the bottom row, then reads right to left across the bottom row, then right to left across the row above that, then right to left across the row above that, etc. This reading order is illustrated at the right of the figure below.

By default, we will use the standard reading order in this paper.

Example 3.12. Let $k=3$ and $n=7$, and let $\lambda=(4,3,1)$. The standard reading order is shown at the right of the figure below.

\begin{tabular}{|l|l|l|l|}
\hline$s_{4}$ & $s_{3}$ & $s_{2}$ & $s_{1}$ \\
\hline$s_{5}$ & $s_{4}$ & $s_{3}$ & \multicolumn{1}{|l}{} \\
\cline { 1 - 2 }$s_{6}$ & \multicolumn{2}{|l}{} \\
\cline { 1 - 1 } &
\end{tabular}

\begin{tabular}{|l|l|l|l|}
\hline 8 & 7 & 6 & 5 \\
\cline { 1 - 2 } 4 & 3 & 2 & \multicolumn{1}{|c}{} \\
\cline { 1 - 2 } 1 & \multicolumn{2}{|c}{} \\
\cline { 1 - 1 } & & \multicolumn{2}{|c}{}
\end{tabular}

Then the following diagrams are Go-diagrams of shape $\lambda$.
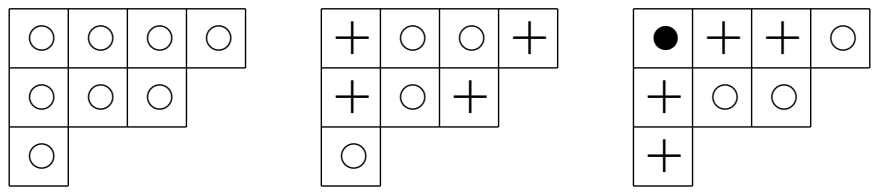

They correspond to the expressions

$$
s_{6} s_{3} s_{4} s_{5} s_{1} s_{2} s_{3} s_{4}, \quad s_{6} 1 s_{4} 11 s_{2} s_{3} 1, \quad 1 s_{3} s_{4} 1 s_{1} 11 s_{4} .
$$

The first and second are positive distinguished subexpressions (PDS's), and the third one is a distinguished subexpression (but not a PDS).

Note that the following diagram of shape $\lambda$ is not a Go-diagram. It corresponds to the word $11 s_{4} 1 s_{1} s_{2} 11$, which is not distinguished.

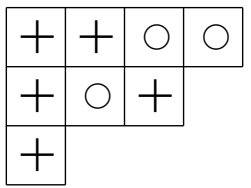


Remark 3.13. The Go-diagrams associated to PDS's are in bijection with J-diagrams; see [Kodama and Williams 2013, Section 4]. Note that the Go-diagram associated to a PDS contains only pluses and white stones. This is precisely a J-diagram.

If we choose a reading order of $\lambda_{w}$, then we will also associate to a Go-diagram of shape $\lambda_{w}$ a labeled Go-diagram, as defined below. Equivalently, a labeled Go-diagram is associated to a pair $(\boldsymbol{v}, \boldsymbol{w})$.

Definition 3.14 [Kodama and Williams 2013, Definition 4.15]. Given a reading order of $\lambda_{w}$ and a Go-diagram of shape $\lambda_{w}$, we obtain a labeled Go-diagram by replacing each $\bigcirc$ with a 1 , replacing each box $b$ containing a $\bullet$ with $\mathrm{a}-1$ and an $m_{i}$, and replacing each box $b$ containing a + by a $p_{i}$, where the subscript $i$ corresponds to the label of $b$ inherited from the reading order.

The labeled Go-diagrams corresponding to the examples above using the standard reading order are:

\begin{tabular}{|l|l|l|l|}
\hline 1 & 1 & 1 & 1 \\
\cline { 1 - 2 } 1 & 1 & 1 & \multicolumn{1}{|c}{} \\
\cline { 1 - 2 } 1 & \multicolumn{2}{|c}{} \\
\cline { 1 - 2 } & & \multicolumn{2}{|c}{}
\end{tabular}

\begin{tabular}{|c|c|c|c|}
\hline$p_{8}$ & 1 & 1 & $p_{5}$ \\
\cline { 1 - 2 }$p_{4}$ & 1 & $p_{2}$ & \multicolumn{1}{|c}{} \\
\cline { 1 - 2 } 1 & \multicolumn{2}{|c}{} \\
\cline { 1 - 2 } &
\end{tabular}

\begin{tabular}{|c|c|c|c|}
\cline { 1 - 2 }$-1 / m_{8}$ & $p_{7}$ & $p_{6}$ & 1 \\
\cline { 1 - 2 }$p_{4}$ & 1 & 1 & \multicolumn{1}{|c}{} \\
\cline { 1 - 1 }$p_{1}$ & \multicolumn{2}{|c}{} \\
\cline { 1 - 1 } & & \multicolumn{2}{|c}{}
\end{tabular}

3D. The main result. To state the main result, we now consider Go-diagrams (not arbitrary diagrams), the corresponding networks (Go-networks), and the corresponding weight matrices.

Definition 3.15. Let $D$ be a Go-diagram contained in a $k \times(n-k)$ rectangle. We define a subset $\mathscr{R}_{D}$ of the Grassmannian $\mathrm{Gr}_{k, n}$ by letting each variable $a_{i}$ of the weight matrix (Definition 3.3) range over all nonzero elements $\mathbb{K}^{*}$, and letting each variable $c_{i}$ of the weight matrix range over all elements $\mathbb{K}$. We call $\mathscr{R}_{D}$ the network component associated to $D$.

Theorem 3.16. Let $D$ be a Go-diagram contained in a $k \times(n-k)$ rectangle. Suppose that $D$ has $t$ pluses and $u$ black stones. Then $\mathscr{R}_{D}$ is isomorphic to $\left(\mathbb{K}^{*}\right)^{t} \times \mathbb{K}^{u}$. Furthermore, $\mathrm{Gr}_{k, n}$ is the disjoint union of the network components $\mathscr{R}_{D}$, as $D$ ranges over all Go-diagrams contained in a $k \times(n-k)$ rectangle. In other words, each point in the Grassmannian $\mathrm{Gr}_{k, n}$ can be represented uniquely by a weighted network associated to a Go-diagram.

A more refined version of Theorem 3.16 is given in Corollary 6.9.

Corollary 3.17. Every $k \times \ell$ matrix $M$ can be represented by a unique weighted network associated to a Go-diagram contained in a $k \times \ell$ rectangle. 
Proof. Let $n=k+\ell$. Suppose $M=\left(m_{i, j}\right)$ is a $k \times(n-k)$ matrix. Let $A(M)=\left(a_{i, j}\right)$ be the full rank $k \times n$ matrix with an identity submatrix in the first $k$ columns and the remaining columns given by

$$
a_{i, j+k}=(-1)^{i+1} m_{n+1-i, j} .
$$

Then $A(M)$ represents an element in the Grassmannian $\mathrm{Gr}_{k, n}$, so Theorem 3.16 applies. The minors of $M$ are in bijection with the $k \times k$ minors of $A(M)$, so if $A(M)$ is represented by the network $N$, we see that $m_{i, j}$ enumerates paths from the boundary source $i$ to the boundary vertex $j$ in $N$.

We will prove Theorem 3.16 by showing that each network component $\mathscr{R}_{D}$ from a Go-diagram coincides with a (projected) Deodhar component $\mathscr{P}_{\boldsymbol{v}, \boldsymbol{w}}$ in the Grassmannian. (Therefore we may refer to each $\mathscr{R}_{D}$ as a Deodhar component.) More specifically, such Deodhar components have parametrizations due to Marsh and Rietsch [2004], and we will show that after an invertible transformation of variables, our network parametrizations coincide with theirs.

\section{The Deodhar decomposition of the Grassmannian}

In this section we review Deodhar's decomposition of the flag variety $G / B$ [Deodhar 1985], and the parametrizations of the components due to [Marsh and Rietsch 2004]. The Deodhar decomposition of the Grassmannian is obtained by projecting the Deodhar decomposition of $G / B$ to the Grassmannian [Deodhar 1987].

4A. The flag variety. Let $\mathbb{K}$ be a field, and let $G$ denote the special linear group $\mathrm{SL}_{n}=\mathrm{SL}_{n}(\mathbb{K})$. Fix a maximal torus $T$ and opposite Borel subgroups $B^{+}$and $B^{-}$; thus $T, B^{+}, B^{-}$consist respectively of the diagonal, upper-triangular, and lowertriangular matrices in $\mathrm{SL}_{n}$. Let $U^{+}$and $U^{-}$be the unipotent radicals of $B^{+}$and $B^{-}$; these are the subgroups of upper-triangular and lower-triangular matrices with 1's on the diagonals. For each $1 \leq i \leq n-1$ we have a homomorphism $\phi_{i}: \mathrm{SL}_{2} \rightarrow \mathrm{SL}_{n}$ such that

$$
\phi_{i}\left(\begin{array}{ll}
a & b \\
c & d
\end{array}\right)=\left(\begin{array}{llllll}
1 & & & & & \\
& \ddots & & & & \\
& & a & b & & \\
& & c & d & & \\
& & & & \ddots & 1
\end{array}\right) \in \mathrm{SL}_{n}
$$

that is, $\phi_{i}$ replaces a $2 \times 2$ block of the identity matrix with $\left(\begin{array}{ll}a & b \\ c & d\end{array}\right)$. Here $a$ is at the $(i+1)$-st diagonal entry counting from the southeast corner. (Correspondingly, we will label the rows of such a matrix from bottom to top, and the columns of such a 
matrix from right to left.) We have 1-parameter subgroups of $G$ defined by

$$
x_{i}(m)=\phi_{i}\left(\begin{array}{cc}
1 & m \\
0 & 1
\end{array}\right) \quad \text { and } \quad y_{i}(m)=\phi_{i}\left(\begin{array}{cc}
1 & 0 \\
m & 1
\end{array}\right), \quad \text { where } m \in \mathbb{K} \text {. }
$$

Let $W$ denote the Weyl group $N_{G}(T) / T$, where $N_{G}(T)$ is the normalizer of $T$. The simple reflections $s_{i} \in W$ are given by $s_{i}:=\dot{s_{i}} T$, where $\dot{s_{i}}:=\phi_{i}\left(\begin{array}{rr}0 & -1 \\ 1 & 0\end{array}\right)$ and any $w \in W$ can be expressed as a product $w=s_{i_{1}} s_{i_{2}} \cdots s_{i_{\ell}}$ with $\ell=\ell(w)$ factors. We set $\dot{w}=\dot{s}_{i_{1}} \dot{s}_{i_{2}} \cdots \dot{s}_{i_{\ell}}$. In our setting $W$ is isomorphic to $\mathfrak{S}_{n}$, the symmetric group on $n$ letters, and $s_{i}$ corresponds to the transposition exchanging $i$ and $i+1$.

We can identify the flag variety $G / B$ with the variety $\mathscr{B}$ of Borel subgroups via

$$
g B \longleftrightarrow g \cdot B^{+}:=g B^{+} g^{-1} .
$$

We have two opposite Bruhat decompositions of $\mathscr{B}$ :

$$
\mathscr{B}=\bigsqcup_{w \in W} B^{+} \dot{w} \cdot B^{+}=\bigsqcup_{v \in W} B^{-} \dot{v} \cdot B^{+} .
$$

We define the intersection of opposite Bruhat cells

$$
\mathscr{R}_{v, w}:=B^{+} \dot{w} \cdot B^{+} \cap B^{-} \dot{v} \cdot B^{+},
$$

which is nonempty precisely when $v \leq w$. The strata $\mathscr{R}_{v, w}$ are often called Richardson varieties.

4B. Deodhar components in the flag variety. We now describe the Deodhar decomposition of the flag variety. Marsh and Rietsch [2004] gave explicit parametrizations for each Deodhar component, identifying each one with a subset in the group.

Definition 4.1 [Marsh and Rietsch 2004, Definition 5.1]. Let $\boldsymbol{w}=s_{i_{1}} \cdots s_{i_{m}}$ be a reduced expression for $w$, and let $\boldsymbol{v}$ be a distinguished subexpression. Define a subset $G_{v, w}$ in $G$ by

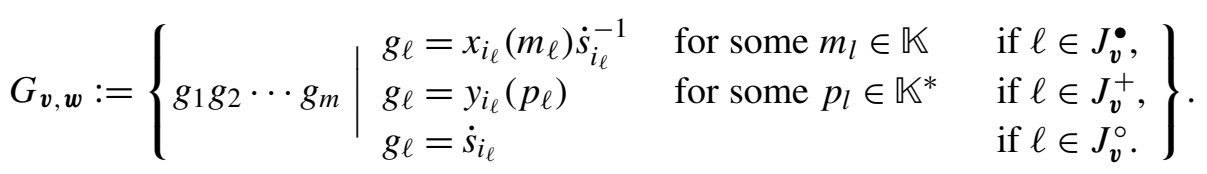

There is an obvious map $\left(\mathbb{K}^{*}\right)^{\left|J_{v}^{+}\right|} \times \mathbb{K}^{\left|J_{v}^{*}\right|} \rightarrow G_{v, w}$ defined by the parameters $p_{\ell}$ and $m_{\ell}$. For $v=w=1$ we define $G_{\boldsymbol{v}, \boldsymbol{w}}=\{1\}$.

Example 4.2. Let $W=\mathfrak{S}_{8}, \boldsymbol{w}=s_{6} S_{7} S_{4} s_{5} s_{6} s_{3} s_{4} s_{5} s_{1} s_{2} s_{3} s_{4}$ and $\boldsymbol{v}=s_{6} 1 s_{4} 1 s_{6} 1 s_{4} 11111$. This is the distinguished expression $v$ encoded by the diagram from Figure 3 (which is a Go-diagram). Then the corresponding element $g \in G_{\boldsymbol{v}, \boldsymbol{w}}$ (the MR-matrix) is 
given by

$$
\begin{aligned}
g=\dot{s}_{6} y_{7}\left(p_{2}\right) \dot{s}_{4} y_{5}\left(p_{4}\right) x_{6}\left(m_{5}\right) \dot{s}_{6}^{-1} y_{3}\left(p_{6}\right) x_{4}\left(m_{7}\right) \dot{s}_{4}^{-1} \\
\cdot y_{5}\left(p_{8}\right) y_{1}\left(p_{9}\right) y_{2}\left(p_{10}\right) y_{3}\left(p_{11}\right) y_{4}\left(p_{12}\right),
\end{aligned}
$$

which is

$$
\left(\begin{array}{cccccccc}
1 & 0 & 0 & 0 & 0 & 0 & 0 & 0 \\
0 & 1 & 0 & 0 & 0 & 0 & 0 & 0 \\
p_{2} & -m_{5} & 1 & 0 & 0 & 0 & 0 & 0 \\
0 & 0 & p_{8} & 1 & 0 & 0 & 0 & 0 \\
0 & -p_{4} & -m_{7} p_{8} & -m_{7}+p_{12} & 1 & 0 & 0 & 0 \\
0 & 0 & -p_{6} p_{8} & -p_{6}+p_{11} p_{12} & p_{11} & 1 & 0 & 0 \\
0 & 0 & 0 & p_{10} p_{11} p_{12} & p_{10} p_{11} & p_{10} & 1 & 0 \\
0 & 0 & 0 & p_{9} p_{10} p_{11} p_{12} & p_{9} p_{10} p_{11} & p_{9} p_{10} & p_{9} & 1
\end{array}\right) .
$$

The following result gives an explicit parametrization for the Deodhar component $\mathscr{R}_{\boldsymbol{v}, \boldsymbol{w}}$. We use Proposition 4.3 as the definition of $\mathscr{R}_{\boldsymbol{v}, \boldsymbol{w}}$.

Proposition 4.3 [Marsh and Rietsch 2004, Proposition 5.2]. The map

$$
\left(\mathbb{K}^{*}\right)^{\left|J_{v}^{+}\right|} \times \mathbb{K}^{\left|J_{v}^{*}\right|} \rightarrow G_{\boldsymbol{v}, \boldsymbol{w}}
$$

from Definition 4.1 is an isomorphism. The map $g \mapsto g \cdot B^{+}$defines an isomorphism

$$
G_{v, w} \stackrel{\sim}{\longrightarrow} \mathscr{R}_{\boldsymbol{v}, \boldsymbol{w}}
$$

between the subset $G_{\boldsymbol{v}, \boldsymbol{w}}$ of the group and the Deodhar component $\mathscr{R}_{\boldsymbol{v}, \boldsymbol{w}}$ in $G / B$.

Suppose that for each $w \in W$ we choose a reduced expression $\boldsymbol{w}$ for $w$. Then it follows from [Deodhar 1985] and [Marsh and Rietsch 2004, Section 4.4] that

$$
\mathscr{R}_{v, w}=\bigsqcup_{\boldsymbol{v} \prec \boldsymbol{w}} \mathscr{R}_{\boldsymbol{v}, \boldsymbol{w}} \quad \text { and } \quad G / B=\bigsqcup_{w \in W}\left(\bigsqcup_{\boldsymbol{v} \prec \boldsymbol{w}} \mathscr{R}_{\boldsymbol{v}, \boldsymbol{w}}\right) \text {, }
$$

where in the first sum $v$ ranges over all distinguished subexpressions for $v$ in $w$, and in the second sum $\boldsymbol{v}$ ranges over all distinguished subexpressions of $\boldsymbol{w}$. These two decompositions are called the Deodhar decompositions of $\mathscr{R}_{v, w}$ and $G / B$.

Remark 4.4. Although the Deodhar decomposition of $\mathscr{R}_{v, w}$ depends on the choice $\boldsymbol{w}$ of reduced expression for $w$, its projection to the Grassmannian does not depend on $\boldsymbol{w}$ [Kodama and Williams 2013, Proposition 4.16].

4C. Projections of Deodhar components to the Grassmannian. Following [Kodama and Williams 2013], we now consider the projection of the Deodhar decomposition to the Grassmannian $\mathrm{Gr}_{k, n}$ for $k<n$. Given the permutation 


$$
w=(w(1), w(2), \ldots, w(n)) \in W^{k},
$$

we let

$$
I(w):=\{w(n-k+1), w(n-k+2), \ldots, w(n)\},
$$

a $k$-element subset of $[n]$. The map $I$ gives a bijection between $W^{k}$ and $k$-element subsets of $[n]$.

Let $\pi_{k}: G / B \rightarrow \mathrm{Gr}_{k, n}$ be the projection from the flag variety to the Grassmannian; this is an isomorphism on each $\mathscr{R}_{v, w}$. For each $w \in W^{k}$ and $v \leq w$, define $\mathscr{P}_{v, w}=\pi_{k}\left(\mathscr{R}_{v, w}\right)$. Then by [Lusztig 1998] we have a decomposition

$$
\mathrm{Gr}_{k, n}=\bigsqcup_{w \in W^{k}}\left(\bigsqcup_{v \leq w} \mathscr{P}_{v, w}\right)
$$

For each reduced decomposition $\boldsymbol{w}$ for $w \in W^{k}$, and each $\boldsymbol{v} \prec \boldsymbol{w}$, we define $\mathscr{P}_{\boldsymbol{v}, \boldsymbol{w}}=\pi_{k}\left(\mathscr{R}_{\boldsymbol{v}, \boldsymbol{w}}\right)$. Now if for each $w \in W^{k}$ we choose a reduced decomposition $\boldsymbol{w}$, then we have

$$
\mathscr{P}_{v, w}=\bigsqcup_{\boldsymbol{v}<\boldsymbol{w}} \mathscr{P}_{\boldsymbol{v}, \boldsymbol{w}} \quad \text { and } \quad \mathrm{Gr}_{k, n}=\bigsqcup_{w \in W^{k}}\left(\bigsqcup_{\boldsymbol{v}<\boldsymbol{w}} \mathscr{P}_{\boldsymbol{v}, \boldsymbol{w}}\right),
$$

where in the first sum $v$ ranges over all distinguished subexpressions for $v$ in $w$, and in the second sum $\boldsymbol{v}$ ranges over all distinguished subexpressions of $\boldsymbol{w}$.

Proposition 4.3 gives a concrete way to think about the projected Deodhar components $\mathscr{P}_{\boldsymbol{v}, \boldsymbol{w}}$. The projection $\pi_{k}: G / B \rightarrow \mathrm{Gr}_{k, n}$ maps $g \cdot B^{+} \in R_{\boldsymbol{v}, \boldsymbol{w}}$ (where $g \in G_{\boldsymbol{v}, \boldsymbol{w}}$ ) to the span of the leftmost $k$ columns of $g$. More specifically, it maps

$$
g=\left(\begin{array}{ccccc}
g_{n, n} & \ldots & g_{n, n-k+1} & \ldots & g_{n, 1} \\
\vdots & & \vdots & & \vdots \\
g_{1, n} & \ldots & g_{1, n-k+1} & \ldots & g_{1,1}
\end{array}\right) \rightarrow M=\left(\begin{array}{ccc}
g_{1, n-k+1} & \ldots & g_{n, n-k+1} \\
\vdots & & \vdots \\
g_{1, n} & \ldots & g_{n, n}
\end{array}\right)
$$

We call the resulting $k \times n$ matrix $M=\left(M_{s t}\right)$ the MR-matrix. To simplify the notation later, we will label its rows from top to bottom by $i_{1}, i_{2}, \ldots, i_{k}$, where $\left\{i_{1}<\cdots<i_{k}\right\}=I(w)$.

Remark 4.5. Recall from Section $3 \mathrm{C}$ that in the Grassmannian setting (that is, $W_{k}=\left\langle s_{1}, s_{2}, \ldots, \hat{s}_{n-k}, \ldots, s_{n-1}\right\rangle$ is a parabolic subgroup of $\left.W=\mathfrak{S}_{n}\right)$, the distinguished subexpressions of $W^{k}$ are in bijection with Go-diagrams. Therefore each Go-diagram gives rise to an MR-matrix.

Example 4.6. We continue Example 4.2. Note that $w \in W^{k}$, where $k=2$. Then the map $\pi_{2}: G_{v, w} \rightarrow \mathrm{Gr}_{2,5}$ is given by 


$$
\begin{aligned}
g & =\left(\begin{array}{cccccccc}
1 & 0 & 0 & 0 & 0 & 0 & 0 & 0 \\
0 & 1 & 0 & 0 & 0 & 0 & 0 & 0 \\
p_{2}-m_{5} & 1 & 0 & 0 & 0 & 0 & 0 \\
0 & 0 & p_{8} & 1 & 0 & 0 & 0 & 0 \\
0 & -p_{4} & -m_{7} p_{8} & -m_{7}+p_{12} & 1 & 0 & 0 & 0 \\
0 & 0 & -p_{6} p_{8} & -p_{6}+p_{11} p_{12} & p_{11} & 1 & 0 & 0 \\
0 & 0 & 0 & p_{10} p_{11} p_{12} & p_{10} p_{11} & p_{10} & 1 & 0 \\
0 & 0 & 0 & p_{9} p_{10} p_{11} p_{12} & p_{9} p_{10} p_{11} & p_{9} p_{10} & p_{9} & 1
\end{array}\right) \\
M & =\left(\begin{array}{ccccccccc}
p_{9} p_{10} p_{11} p_{12} & p_{10} p_{11} p_{12} & -p_{6}+p_{11} p_{12} & -m_{7}+p_{12} & 1 & 0 & 0 & \\
0 & 0 & -p_{6} p_{8} & -m_{7} p_{8} & p_{8} & 1 & 0 & 0 \\
0 & 0 & 0 & -p_{4} & 0 & -m_{5} & 1 & 0 \\
0 & 0 & 0 & 0 & 0 & p_{2} & 0 & 1
\end{array}\right)
\end{aligned}
$$

We label the rows of $M$ from top to bottom by the index set $\{1,3,4,6\}$, and the columns from left to right by the index set $\{1,2, \ldots, 8\}$, so for example $M_{34}=-m_{7} p_{8}$.

The following lemma is a consequence of Section 5.1 (and Corollary 5.8 in particular) in [Kodama and Williams 2013].

Lemma 4.7. Let $M=M_{D}$ be the MR-matrix associated to the diagram $D$. The leftmost nonzero entry in row $i_{\ell}$ of $M$ is in column $i_{\ell}$. Furthermore, that entry is equal to $(-1)^{b} \prod p_{i}$, where $b$ is the number of black stones in the row $i_{\ell}$ of $D$, and the product is over all boxes in the row $i_{\ell}$ of the labeled Go-diagram of $D$ containing $a p_{i}$.

\section{Formulas for entries of the MR-matrices}

In this section we consider arbitrary diagrams (not necessarily Go-diagrams) contained in a $k \times(n-k)$ rectangle and the corresponding MR-matrices, obtained by multiplying factors $\dot{s}_{i}, y_{i}\left(p_{j}\right), x_{i}\left(m_{j}\right) \dot{s}_{i}^{-1}$ as specified by the filling of the diagram, and then projecting the resulting $n \times n$ matrix to a $k \times n$ matrix. We will give formulas for the entries of the MR-matrices in terms of pseudopaths in the corresponding network. For the purpose of giving this formula, we will replace weights $a_{i}$ and $c_{j}$ on the edges of the network by weights $p_{i}$ and $m_{j}$.

Recall that if $D$ is a diagram, its network $N_{D}$ has three types of vertices: +-vertices, $\bullet$-vertices, and boundary vertices. A step on a network is an edge between two vertices. Let $W$ denote a single step west, $S$ denote a single step south, and $E$ denote either a single step east, or an east-west combination step consisting of a step east, followed by a step west ending at a $\bullet$. Let $A^{*}$ indicate 0 or more instances of a step of type $A$. 
Definition 5.1. A pseudopath $\widetilde{P}$ on a network is a path on the (undirected version of the) network such that:

- it starts and ends at two different boundary vertices, or else is the empty path from a boundary vertex to itself;

- it does not cross the same edge twice;

- its sequence of steps (for a nonempty path) has the form

$$
W W^{*} S\left(E E^{*} S\right)^{*} E^{*} \text {. }
$$

In particular, a pseudopath may not take two consecutive steps south.

Definition 5.2. The weight $w(\widetilde{P})$ of a pseudopath $\widetilde{P}$ in a network is a Laurent monomial in $p_{i}$ 's and $m_{j}$ 's, which is obtained by multiplying the following terms:

- $1 / p_{i}$ for every step west along an edge weighted $p_{i}$;

- $p_{i}$ for every step east along an edge weighted $p_{i}$ which is preceded by a step east;

- $m_{j}$ for every step west along an edge weighted $m_{j}$;

- $(-1)^{b+w}$, where $b$ (respectively $w$ ) is the number of black (resp. white) stones that the pseudopath skips over in the horizontal (resp. vertical) direction when we superimpose the Go-diagram onto the network.

Example 5.3. In Figure 5, there are two pseudopaths from 1 to 4 , with weights $1 /\left(p_{9} p_{10} p_{11}\right)$ and $-m_{7} /\left(p_{9} p_{10} p_{11} p_{12}\right)$, and there is one pseudopath from 1 to 5 , with weight $1 /\left(p_{9} p_{10} p_{11} p_{12}\right)$.

Definition 5.4. If $M$ is an MR-matrix, we will let $\tilde{M}$ denote the matrix obtained from $M$ by rescaling rows so that the leftmost nonzero entry in each row is 1 .

Definition 5.5. If $D$ is a diagram contained in a $k \times(n-k)$ rectangle, then we let $i_{1}<i_{2}<\cdots<i_{k}$ denote the labels of the sources in the corresponding network. If
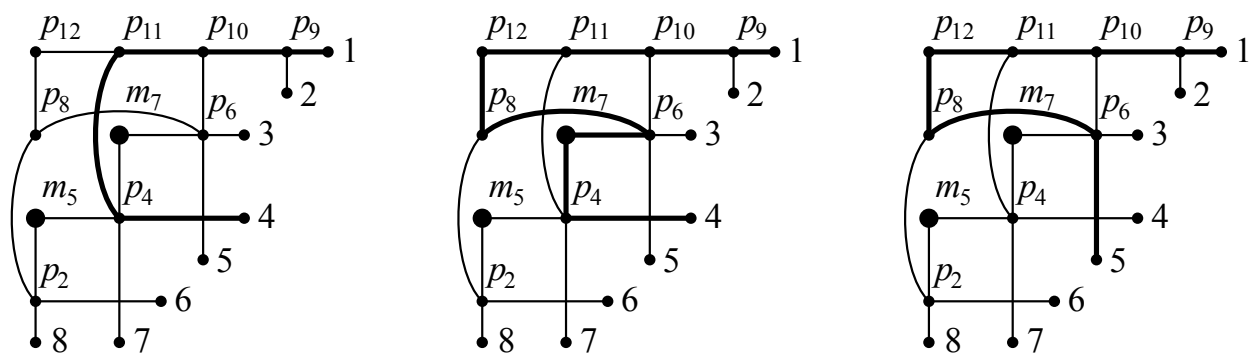

Figure 5. The two pseudopaths from 1 to 4 and the unique pseudopath from 1 to 5 , indicated in bold. Note that the pseudopath in the middle figure contains an east-west combination step. 
$M$ and $\tilde{M}$ are the corresponding $k \times n$ MR and rescaled MR-matrices associated to $D$, then we will index their rows by $i_{1}, \ldots, i_{k}$ from top to bottom and their columns by $1,2, \ldots, n$ from left to right.

Theorem 5.6. Let $D$ be a diagram contained in a $k \times(n-k)$ rectangle, and let $\widetilde{M}=\left(\widetilde{M}_{s t}\right)$ be the corresponding $k \times n$ rescaled MR-matrix. Then

$$
\tilde{M}_{s t}=\sum_{\widetilde{P}} w(\widetilde{P}),
$$

where the sum is over all pseudopaths from the source s to the boundary vertex $t$ in the network.

Theorem 5.6 will follow from Theorem 5.10 and Lemma 5.11.

Example 5.7. The MR-matrix $M$ from Example 4.6 corresponds to the network from Figure 5. The rows of $M$ are indexed by 1, 3, 4, 6 from top to bottom. Note that after we rescale the rows of $M$, obtaining $\tilde{M}$, we have

$$
\tilde{M}_{14}=\frac{1}{p_{9} p_{10} p_{11}}-\frac{m_{7}}{p_{9} p_{10} p_{11} p_{12}} \quad \text { and } \quad \tilde{M}_{15}=\frac{1}{p_{9} p_{10} p_{11} p_{12}} .
$$

This agrees with our pseudopath computation from Example 5.3.

Next we will give a formula for entries of MR-matrices, in terms of pseudopaths in modified networks.

Definition 5.8. Given a network $N_{D}$ with $k$ sources labeled $i_{1}, \ldots, i_{k}$ and $n$ boundary vertices, we obtain from it a corresponding modified network $N_{D}^{\prime}$ by:

- adding $k$ new boundary vertices to the left of $N_{D}$, labeled $i_{1}^{\prime}, \ldots, i_{k}^{\prime}$ from top to bottom;

- adding a new horizontal edge which connects $i_{j}^{\prime}$ to the nearest vertex of the network to its right.

See Figure 6 for the modified network associated to the network from Figure 5.

Definition 5.9. A pseudopath $P$ on a modified network is a path on the modified network which:

- starts at one of the boundary vertices labeled $i_{1}^{\prime}, \ldots, i_{k}^{\prime}$, and ends at one of the boundary vertices labeled $1,2, \ldots, n$;

- takes a sequence of steps which has the form

$$
\left(E E^{*} S\right)^{*} E^{*} \text {. }
$$

The arrows in Figure 6 indicate the allowed directions in which a path may travel. The weight of a pseudopath in a modified network is defined the same way as the weight of a pseudopath in a network (see Definition 5.2). Note that since 


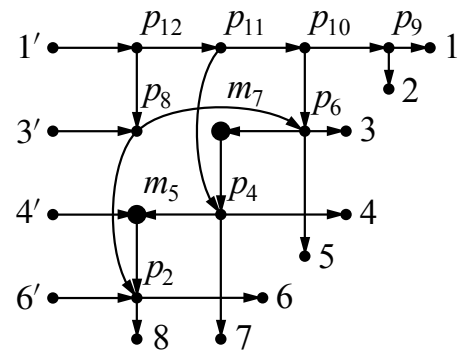

Figure 6. Example of a modified network.

a pseudopath in a modified network does not contain steps west along edges weighted $p_{i}$, its weight is a monomial (not a Laurent monomial).

Theorem 5.10. Let $D$ be a diagram contained in a $k \times(n-k)$ rectangle, and let $M=\left(M_{s t}\right)$ be the corresponding $k \times n$ MR-matrix. Then

$$
M_{s t}=\sum_{P} w(P),
$$

where the sum is over all pseudopaths in the modified network from the boundary vertex $s^{\prime}$ to the boundary vertex $t$.

Lemma 5.11. Theorems 5.6 and 5.10 are equivalent.

Proof. There is an obvious bijection between pseudopaths in a network starting at boundary vertex $s$, and pseudopaths in the corresponding modified network starting at boundary vertex $s^{\prime}$. The weights of the corresponding pseudopaths are the same except for a factor of $(-1)^{b} \prod p_{i}$, where $b$ is the number of $\bullet$-vertices in row $s$ of the network, and the $p_{i}$ 's range over all edge weights in row $s$.

On the other hand, Lemma 4.7 implies that the leftmost nonzero entry of row $s$ of the MR-matrix $M$ is precisely the quantity $(-1)^{b} \prod p_{i}$ above. Therefore Theorems 5.6 and 5.10 are equivalent.

By Lemma 5.11, in order to prove Theorem 5.6 it suffices to prove Theorem 5.10. Our strategy for proving Theorem 5.10 will be to interpret entries of the MR-matrix in terms of paths in a chip network, and then construct a weight-preserving bijection between these paths and between pseudopaths in the modified network.

Definition 5.12. A chip is one of the three configurations shown in Figure 7. We call the three configurations $y_{i}(p)$ - or $y_{i}$-chips, $s_{i}$-chips, and $x_{i}(m)$ - or $x_{i}$-chips, respectively.

Definition 5.13. A chip network is a concatenation of chips. Note that it has $n$ boundary vertices at the left and $n$ boundary vertices at the right. Let $g$ be any product of factors of the form $y_{i}(p), \dot{s}_{i}$, and $x_{i}(m) \dot{s}_{i}^{-1}$. We associate a chip network 

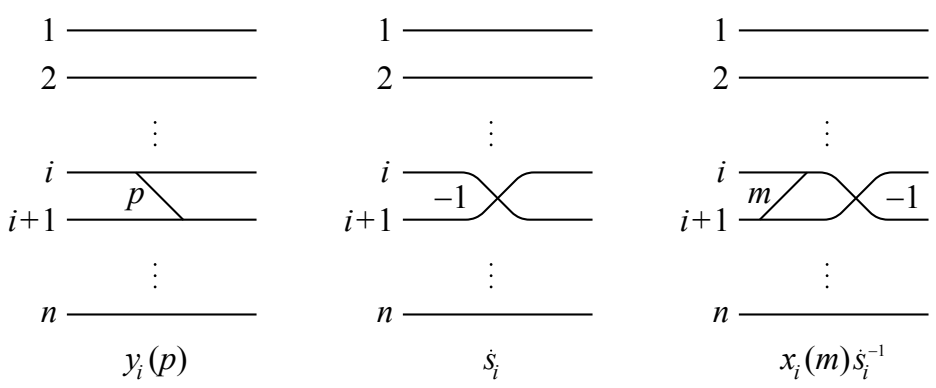

Figure 7. The three types of chips: $y_{i}$-chip (left), $s_{i}$-chip (center), and $x_{i}$-chip (right).

$C_{g}$ to $g$ by concatenating the chips corresponding to the factors of $g$ in the order given by the factorization.

Example 5.14. The chip network $C_{g}$ associated to the product $g$ from (4-1) is shown in Figure 8.

Definition 5.15. A route $Q$ in a chip network is a path in the network whose steps all travel east (or southeast or northeast for slanted edges). The weight $w(Q)$ of such a route is the product of all weights on its edges. To each chip network $C$ we associate a weight matrix $x(C)=x_{i j}$, where $x_{i j}=\sum_{Q} w(Q)$, and the sum is over all routes from the boundary vertex $i$ at the west to the boundary vertex $j$ at the east.

It is simple to verify the following result. Recall our convention from Section 4A that the rows of $g$ are labeled from bottom to top, and the columns of $g$ are labeled from right to left.

Lemma 5.16. Let $g$ be a product of factors of the form $y_{i}(p), \dot{s}_{i}$, and $x_{i}(m) \dot{s}_{i}^{-1}$. Then the weight matrix $x\left(C_{g}\right)$ of the chip network $C_{g}$ associated to $g$ coincides with the matrix $g$.

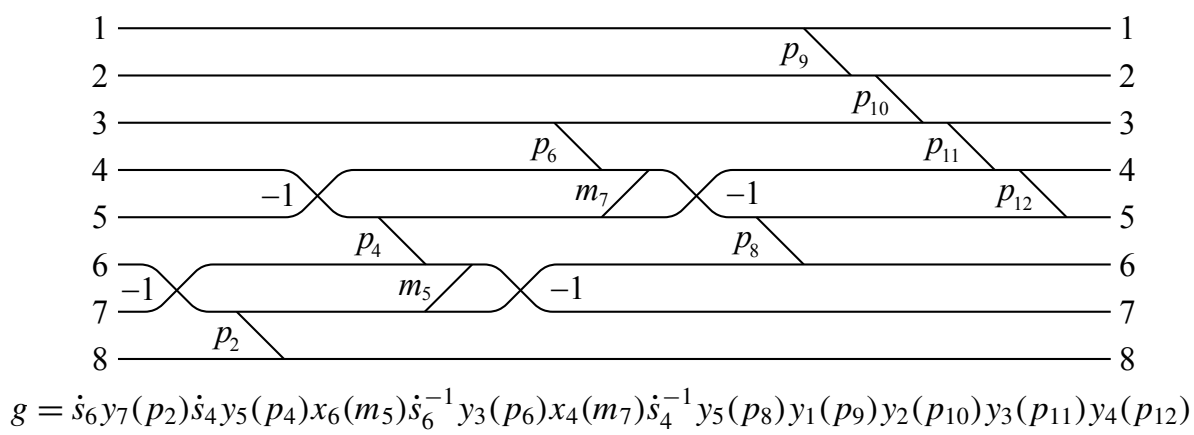

Figure 8. The chip network corresponding to the product $g$ of (4-1). 
Proof of Theorem 5.10. Let $D$ be a diagram contained in a $k \times(n-k)$ rectangle, and $N_{D}^{\prime}$ the corresponding modified network. Let $i_{1}^{\prime}<\cdots<i_{k}^{\prime}$ be the labels of the sources of $N_{D}^{\prime}$. Let $g$ be the product of factors of the form $y_{i}(p), \dot{s}_{i}$, and $x_{i}(m) \dot{s}_{i}^{-1}$ which is encoded by $D$, and let $M$ be the corresponding MR-matrix, whose rows are indexed from top to bottom by $i_{1}, \ldots, i_{k}$. Recall that the projection from $g$ to $M$ switches rows and columns, and the columns labeled $n-k+1, n-k+2, \ldots, n$ in $g$ become rows labeled $i_{1}, i_{2}, \ldots, i_{k}$ in $M$. Therefore, to prove Theorem 5.10 it suffices to prove that for all $1 \leq t \leq n$ and $1 \leq s \leq k$ we have

$$
g_{t, s+(n-k)}=\sum_{P} w(P),
$$

where the sum is over all pseudopaths $P$ from $i_{s}^{\prime}$ to $t$ in the modified network.

By Lemma 5.16, the matrix $g$ coincides with the weight matrix $x\left(C_{g}\right)=\left(x_{s t}\right)$ of the chip network associated to $g$. Therefore by (5-1) it suffices to prove that for all $1 \leq t \leq n$ and $1 \leq s \leq k$ we have

$$
x_{t, s+(n-k)}=\sum_{P} w(P)
$$

where the sum is over all pseudopaths $P$ from $i_{s}^{\prime}$ to $t$ in the modified network.

Recall from Definition 5.15 that $x_{i j}=\sum_{Q} w(Q)$, where the sum is over all routes $Q$ in the chip network from the boundary vertex $i$ at the west to the boundary vertex $j$ at the east. To prove (5-2), we will give a weight-preserving bijection between pseudopaths $P$ in the modified network from $i_{s}^{\prime}$ to $t$, and routes $Q$ in the chip network from the boundary vertex $t$ at the west to the boundary vertex $s+(n-k)$ at the east. More specifically, given a pseudopath $P$, we will examine its sequence of steps from source to sink, and explain how to build the corresponding route $Q$ in the chip network. As illustrated in Figure 9, each step in a pseudopath corresponds to a portion of a route in a chip network. (Note that our bijection will build the route in the chip network from east to west, rather than west to east.)

Recall from Figure 4 that each modified network comes from a diagram, and that every box of a diagram is naturally associated with a simple generator $s_{i}$. Therefore every internal vertex in a modified network is naturally associated with a simple generator $s_{i}$ for some $i$. We will call this the position of the vertex.

Let us consider the various kinds of steps in a pseudopath. Such steps naturally fall into one of the following categories (illustrated in Figure 9):

0. A single step east, which starts at a source and ends at position $s_{i}$.

I. A single step east, which is preceded by a south step and followed by an east or south step. Such a step starts and ends at positions $s_{i}$ and $s_{j}$ (for $i>j$ ), and is labeled by some weight $p$. It may skip over some (positions corresponding to) white and black stones in the Go-diagram. 
(I)

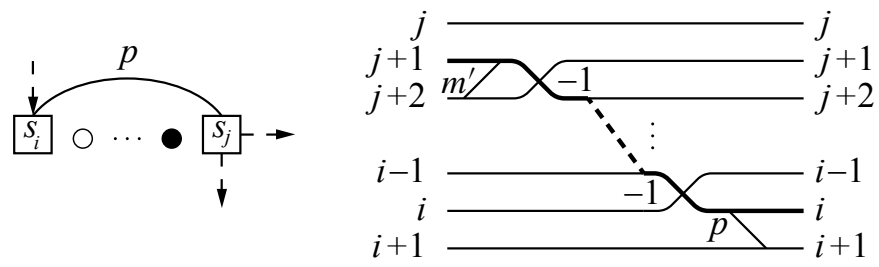

(II)

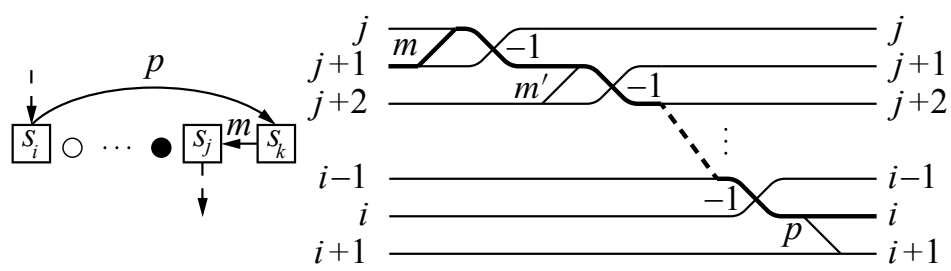

(III)
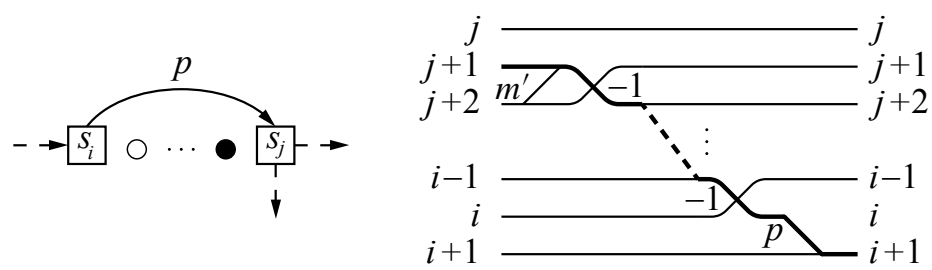

(IV)
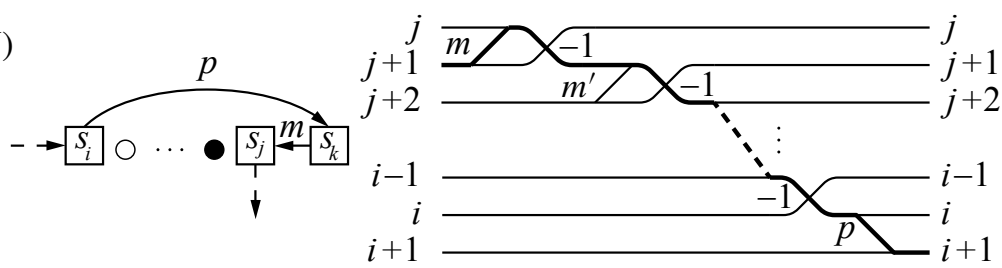

(V)
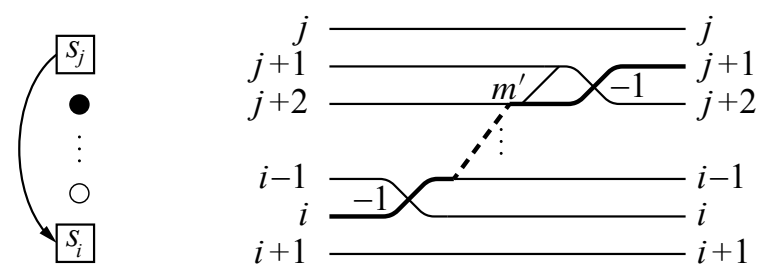

Figure 9. Steps in pseudopaths and their corresponding fragments of the chip network.

II. An east-west combination step, which is preceded by a south step and travels from position $s_{i}$ to $s_{k}$ to $s_{j}$ (where $i>j>k$ ). The two components of such a step are labeled by some weights $p$ and $m$, and may skip over some white and black stones.

III. A single step east, which is preceded by an east step and followed by an east or south step. Such a step starts and ends at positions $s_{i}$ and $s_{j}$ (for $i>j$ ), and is labeled by some weight $p$. It may skip over some white and black stones. 
IV. An east-west combination step, which is preceded by an east step and travels from position $s_{i}$ to $s_{k}$ to $s_{j}$ (where $i>j>k$ ). The two components of such a step are labeled by some weights $p$ and $m$, and may skip over some white and black stones.

V. A south step, which starts and ends at positions $s_{i}$ and $s_{j}$ (for $i<j$ ). Such a step may skip over some white and black stones.

The above steps in a pseudopath correspond to the following portion of a route in a chip network:

0. Start at the boundary vertex $i+1$ at the east of the chip network.

I. Start at level $i$, then travel west straight across the $y_{i}(p)$ chip. For each white or black stone (say in position $s_{\ell}$ ) which lies in between positions $s_{i}$ and $s_{j}$, travel northwest through the corresponding $s_{\ell}$ or $x_{\ell}$-chip, ending at level $j+1$.

II. Start at level $i$, then travel west straight across the $y_{i}(p)$ chip. For each white or black stone (say in position $s_{\ell}$ ) which lies in between positions $s_{i}$ and $s_{j}$, travel northwest through the corresponding $s_{\ell}$ or $x_{\ell}$-chip. Finally, travel along the -1 -edge and then the $m$-edge of the $x_{j}(m)$ chip, ending at level $j+1$.

III. Start at level $i+1$, then travel northwest along the $p$-edge in the $y_{i}(p)$ chip. For each white or black stone (say in position $s_{\ell}$ ) which lies in between positions $s_{i}$ and $s_{j}$, travel northwest through the corresponding $s_{\ell}$ or $x_{\ell}$-chip, ending at level $j+1$.
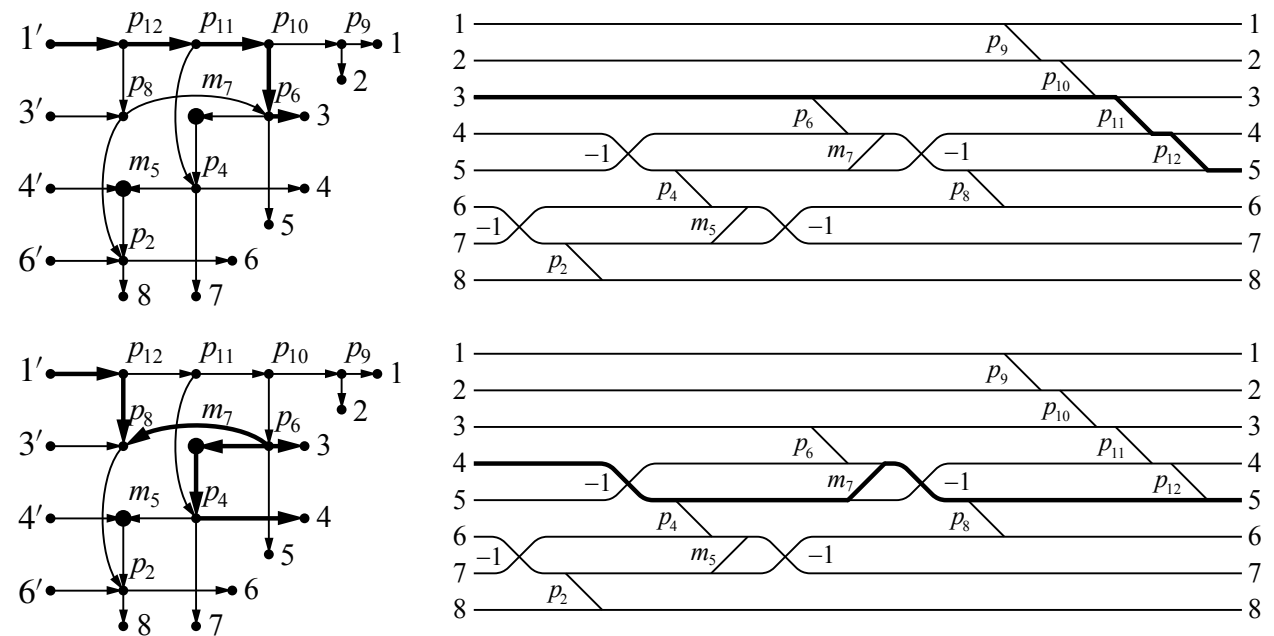

Figure 10. Pseudopaths in the modified network and their corresponding routes in the chip network. 
IV. Start at level $i+1$, then travel northwest along the $p$-edge in the $y_{i}(p)$ chip. For each white or black stone (say in position $s_{\ell}$ ) which lies in between positions $s_{i}$ and $s_{j}$, travel northwest through the corresponding $s_{\ell}$ or $x_{\ell}$-chip. Finally, travel along the -1 -edge and the $m$-edge of the $x_{j}(m)$ chip, ending at level $j+1$.

V. Start at level $i+1$. For each white or black stone (say in position $s_{\ell}$ ) which lies in between positions $s_{i}$ and $s_{j}$, travel southwest through the corresponding $s_{\ell}$ or $x_{\ell}$-chip, ending at level $j$.

It is a straightforward exercise to verify that this map is a bijection between pseudopaths $P$ from $i_{s}^{\prime}$ to $t$ in the modified network, and routes $Q$ between the $t$ vertex at the west and the $s+(n-k)$ vertex at the east in the chip network. Moreover, the weights of $P$ and $Q$ are equal. See Figure 10 for examples of entire pseudopaths and routes.

\section{Proof of the main result}

Let $D$ be a diagram which contains $t$ pluses and $u$ black stones. In Section 6A we will define an isomorphism

$$
\Psi=\Psi_{D}:\left(\mathbb{K}^{*}\right)^{t} \times \mathbb{K}^{u} \rightarrow\left(\mathbb{K}^{*}\right)^{t} \times \mathbb{K}^{u}
$$

which maps each parameter from the weight matrix of the network $N_{D}$ to a Laurent monomial in the parameters used in the MR-matrix $M=M_{D}$. Then in Section 6B we will show that after applying $\Psi$, our network parametrization of the network component $\mathscr{R}_{D}$ coincides with the corresponding MR-parametrization of the projected Deodhar component $\mathscr{P}_{\boldsymbol{v}, \boldsymbol{w}}$. Combining this fact with Proposition 4.3 yields Theorem 3.16.

\section{A. A rational transformation of parameters.}

Definition 6.1. Let $D$ be a diagram, and let $b_{0}$ be a box of $D$ containing a + or Let $b_{1}$ be the nearest box to the right of $b_{0}$ which contains a + (if it exists). Let $R_{t}$ be the set of boxes in the same row as $b_{0}$ which are to the right of $b_{0}$ and left of $b_{1}$. Let $R_{\ell}$ be the set of boxes in the same column as $b_{0}$ and below $b_{0}$. If $b_{1}$ exists, let $R_{r}$ be the set of boxes in the same column as $b_{1}$ and below $b_{1}$ (otherwise $R_{r}=\varnothing$ ). (See Figure 11.) Let $R_{r}^{+}$(resp. $R_{\ell}^{+}$) be the set of boxes in $R_{r}$ (resp. $R_{\ell}$ ) containing a + . Let $R=R_{r} \cup R_{\ell} \cup R_{t}$ and let $R^{\bullet}$ be the set of boxes in $R$ containing a

If $b_{0}$ contains a + , then define

$$
\Psi\left(a_{b_{0}}\right)=\frac{(-1)^{\left|R^{\bullet}\right|} \prod_{b \in R_{r}^{+}} p_{b}}{p_{b_{0}} \prod_{b \in R_{\ell}^{+}} p_{b}} .
$$




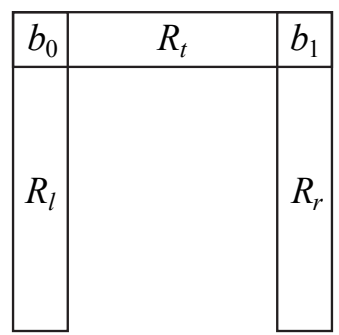

Figure 11. The definition of $b_{0}, b_{1}, R_{t}, R_{\ell}$, and $R_{r}$.

And if $b_{0}$ contains a $\bullet$, then define

$$
\Psi\left(c_{b_{0}}\right)=\frac{m_{b_{0}}(-1)^{\left|R^{\bullet}\right|} \prod_{b \in R_{r}^{+}} p_{b}}{\prod_{b \in R_{\ell}^{+}} p_{b}} .
$$

We also extend the definition of $\Psi$ to all polynomials in the $a_{b}$ 's and $c_{b}$ 's by requiring it to be a ring homomorphism.

Remark 6.2. Clearly $\Psi=\Psi_{D}$ is an isomorphism from $\left(\mathbb{K}^{*}\right)^{t} \times \mathbb{K}^{u}$ to itself.

Example 6.3. Consider the network from Figure 3 (shown again in Figure 12). Then we have $\Psi\left(a_{2}\right)=1 / p_{2}, \Psi\left(a_{4}\right)=1 / p_{4}, \Psi\left(c_{5}\right)=m_{5} / p_{2}, \Psi\left(a_{6}\right)=1 / p_{6}$, $\Psi\left(c_{7}\right)=m_{7} / p_{4}, \Psi\left(a_{8}\right)=1 /\left(p_{2} p_{8}\right), \Psi\left(a_{9}\right)=1 / p_{9}, \Psi\left(a_{10}\right)=1 /\left(p_{6} p_{10}\right), \Psi\left(a_{11}\right)=$ $-p_{6} /\left(p_{4} p_{11}\right)$, and $\Psi\left(a_{12}\right)=p_{4} /\left(p_{2} p_{8} p_{12}\right)$.

From Definition 6.1, one may easily deduce a formula for $\Psi(w(P))$, where $P$ is a path in $N_{D}$. We will state this formula in terms of the Go-diagram. Note that one may identify a path $P$ in $N_{D}$ with a connected sequence $\mathscr{P}$ of boxes in the Go-diagram, where any two adjacent boxes must share a side. We call a box $b$ in $\mathscr{P}$ a corner box if the path $P$ turns from west to south, or from south to west, at the vertex associated to $b$. (Such a box $b$ in $D$ must contain a + or $\bullet$.)

The next result, whose proof is left to the reader, is a simple consequence of Definition 6.1.

Proposition 6.4. Let $P$ be a path in the network $N_{D}$, which we identify with a sequence $\mathscr{P}$ of boxes in the Go-diagram $D$. Among the boxes in $\mathscr{P}$, let $B_{1}$ denote the subset containing $a+$; let $B_{2}$ denote the subset containing $a \bullet$ which are corner boxes of $\mathscr{P}$; and let $B_{3}$ denote the subset containing $a \bullet$ which are not corner boxes of $\mathscr{P}$. Then

$$
\Psi(w(P))=\frac{(-1)^{\left|B_{3}\right|} \prod_{b \in B_{2}} m_{b}}{\prod_{b \in B_{1}} p_{b}} .
$$

Example 6.5. Let $P_{1}$ and $P_{2}$ be the paths shown in Figure 12. Then

$$
\Psi\left(w\left(P_{1}\right)\right)=\Psi\left(a_{9} a_{10} a_{11} c_{5}\right)=\frac{-m_{5}}{p_{2} p_{4} p_{9} p_{10} p_{11}}
$$



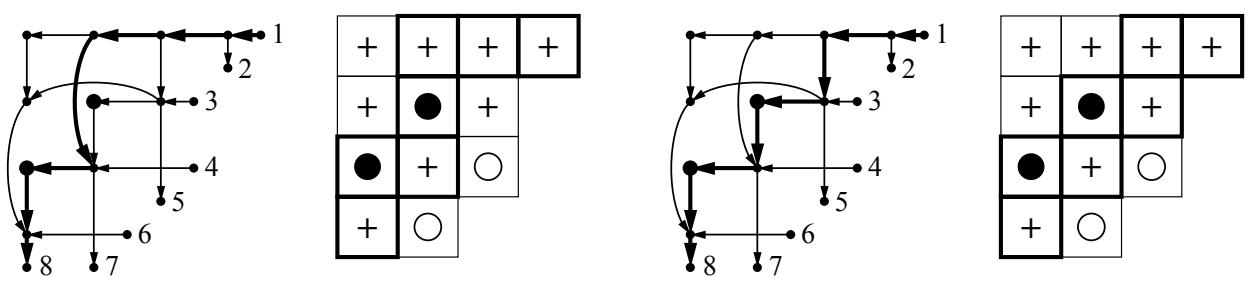

Figure 12. Two paths in $N_{D}$ and their corresponding sequences of boxes in $D$.

and

$$
\Psi\left(w\left(P_{2}\right)\right)=\Psi\left(a_{9} a_{10} c_{5} c_{7}\right)=\frac{m_{5} m_{7}}{p_{2} p_{4} p_{6} p_{9} p_{10}} .
$$

\section{B. Applying row operations to the rescaled MR-matrix.}

Theorem 6.6. Let $D$ be a diagram, let $M=M_{D}$ be the corresponding MR-matrix, and let $L$ be the matrix we obtain by putting $M$ into reduced row-echelon form. Let $W_{D}=\left(W_{i j}\right)$ be the weight matrix associated to $D$, and let $\Psi\left(W_{D}\right)$ be the matrix obtained from $W_{D}$ by applying the rational map $\Psi$ to each entry. Then $\Psi\left(W_{D}\right)=L$. Proof. To prove Theorem 6.6, we start by considering the rescaled MR-matrix $\tilde{M}$. Its rows are indexed by the set $i_{1}, \ldots, i_{k}$, the set of sources of the network $N_{D}$, and the leftmost nonzero entry in every row is a 1. Moreover, by Lemma 4.7, the 1 in row $i_{\ell}$ is located in column $i_{\ell}$. The entries of the reduced row-echelon matrix $L$ obtained from $\widetilde{M}$ are given by the formula

$$
L_{s t}=\tilde{M}_{s t}+\sum_{s<j_{1}<\cdots<j_{r}<t}(-1)^{r} \tilde{M}_{s j_{1}} \tilde{M}_{j_{1} j_{2}} \cdots \tilde{M}_{j_{r} t},
$$

where the sum ranges over all nonempty subsets $\left\{j_{1}, \ldots, j_{r}\right\} \subset\left\{i_{1}, \ldots, i_{k}\right\}$ of sources of the network between $s$ and $t$.

By Theorem 5.6, the entry $\widetilde{M}_{s t}$ equals $\sum_{\widetilde{P}} w(\widetilde{P})$, where the sum is over all pseudopaths in the network $N_{D}$ from the source $s$ to the boundary vertex $t$. Therefore, the right-hand side of (6-2) can be interpreted as a generating function for all concatenations of pseudopaths, where the first pseudopath starts at $s$ and the last pseudopath ends at $t$.

Let us identify a pseudopath with its collection of directed edges. Given a set of pseudopaths on $N_{D}$, we define its signed union to be the union of directed edges that one obtains by taking the multiset of all directed edges in the pseudopaths, and then cancelling pairs which traverse the same edge but in opposite directions. We define the weight of a set of pseudopaths to be the product of the weights of each pseudopath in the set.

Our goal is to show that after cancellation, the only terms which survive on the right-hand side of (6-2) correspond to concatenations of pseudopaths whose signed 

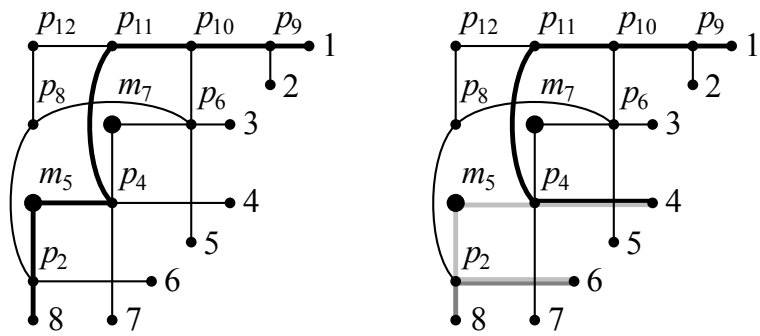

Figure 13. A path in $N_{D}$ and its decomposition into U-turn pseudopaths.

union forms a directed path (and not merely a pseudopath) from $s$ to $t$ in $N_{D}$. This will allow us to relate (6-2) to $W_{s t}$, which is defined as a sum over all paths from $s$ to $t$ in $N_{D}$.

Definition 6.7. A $U$-turn pseudopath in a network is a pseudopath whose sequence of steps has the form $\left(W W^{*} S\right) E^{*}$.

First note that any path $P$ in a network $N_{D}$ has a unique decomposition as a signed union of U-turn pseudopaths. Moreover, the products of the weights of the pseudopaths is precisely the quantity on the right-hand side of (6-1). See Figure 13 for an example of the decomposition into U-turn pseudopaths.

This observation on the decomposition of paths may be generalized to pseudopaths. Consider a pseudopath $\widetilde{P}$ which is not a path, and turns from south to east precisely $q$ times (for $q \geq 1$ ). Then for each $0 \leq r \leq q$, there are $\left(\begin{array}{l}q \\ r\end{array}\right)$ decompositions of $\widetilde{P}$ as a signed union of $r$ pseudopaths. Moreover, each set of pseudopaths forming a decomposition of $\widetilde{P}$ has the same weight. See Figure 14 for an example of all decompositions of a pseudopath as a signed union of pseudopaths. It is easy to check that each decomposition has the same weight.

Note that since all decompositions of a pseudopath have the same weight, and because

$$
\left(\begin{array}{l}
q \\
0
\end{array}\right)-\left(\begin{array}{l}
q \\
1
\end{array}\right)+\left(\begin{array}{l}
q \\
2
\end{array}\right)-\cdots \pm\left(\begin{array}{l}
q \\
q
\end{array}\right)=0 \text { for } q \geq 1
$$
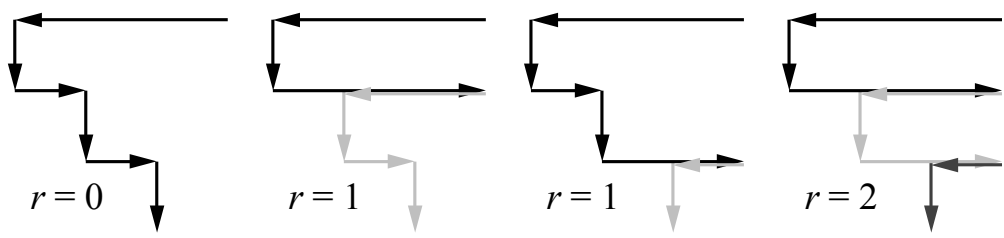

Figure 14. An example of the $2^{q}$ decompositions of a path in $N_{D}$ into pseudopaths. 

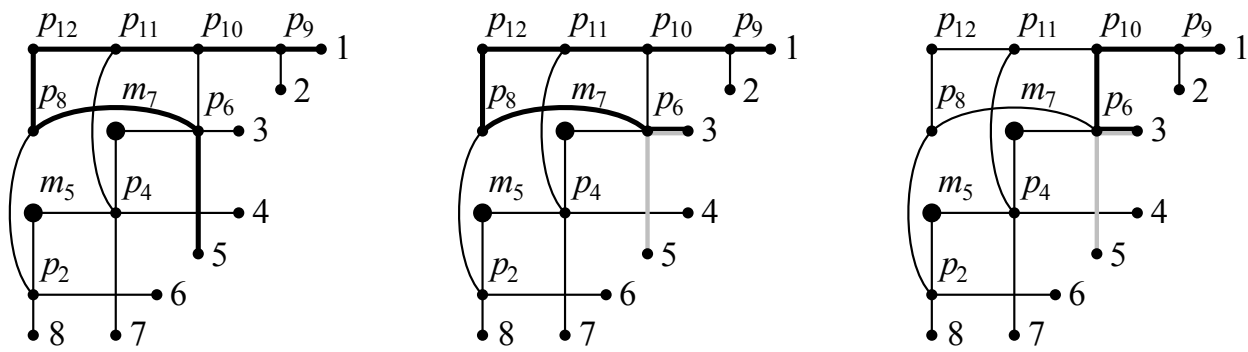

Figure 15. The three possible concatenations of pseudopaths from 1 to 5 .

the net contribution of the corresponding concatenations of pseudopaths in (6-2) is 0 .

More generally, a term on the right-hand side of (6-2) corresponds to a concatenation of pseudopaths (whose signed union may not be a pseudopath). However, just as before, one may decompose the signed union $\widetilde{P}$ in $2^{q}$ ways, where $q$ is the number of times that $\widetilde{P}$ turns from south to east. And again, for $q \geq 1$, the net contribution of the corresponding concatenations of pseudopaths in (6-2) is 0 . Therefore when one interprets the right-hand side of (6-2) as a sum over concatenations of pseudopaths, the only terms that are not cancelled are the terms corresponding to concatenations of pseudopaths whose signed union is a directed path.

Example 6.8. In Figure 15, the left and middle diagrams show a pseudopath from 1 to 5 , and a concatenation of two pseudopaths from 1 to 5, whose weights cancel each other. The right diagram shows a path from 1 to 5 , written as a signed union of two pseudopaths, which will not be cancelled by any other term.

It is a simple exercise to verify that the absolute value of the weight of such a concatenation is precisely the absolute value of the right-hand side of (6-1). We also need to verify that the signs agree. Once we do this, then since $W_{s t}$ is the sum of all weights of paths in $N_{D}$ from $s$ to $t, \Psi\left(W_{s t}\right)$ equals the corresponding term in the expression for $L_{s t}$ in (6-2), so the proof is done.

We now check that the signs agree. More specifically, consider a concatenation of $(r+1)$ pseudopaths whose signed union is a directed path $P$. Using Definition 5.2, the total sign associated to the concatenation of pseudopaths from $(6-2)$ is $(-1)^{r+b+w}$, where $b$ (resp. $w$ ) is the number of black (resp. white) stones that $P$ skips over in the horizontal (resp. vertical) direction. (Note that for the purpose of computing $b$ and $w$, we can count stones skipped by $P$ here, as opposed to the set of pseudopaths whose signed union is $P$.) Meanwhile, using Definition 3.3 and Proposition 6.4, the total sign associated to the directed path $P$ in the expression $\Psi\left(W_{s t}\right)$ is $(-1)^{q+\left|B_{3}\right|}$, where $q$ is the number of sources in the network which are strictly between $s$ and $t$, and $\left|B_{3}\right|$ is the number of noncorner 
black stones in the boxes of the Go-diagram which $P$ traces out. We need to show that $(-1)^{r+b+w}=(-1)^{q+\left|B_{3}\right|}$.

Note that $\left|B_{3}\right|$ is the number of black stones skipped either vertically or horizontally by $P$. Let $b_{v}$ (resp. $w_{v}$ ) denote the number of black stones (resp. white stones) skipped vertically by $P$. Similarly, let $b_{h}$ (resp. $w_{h}$ ) denote the number of black stones (resp. white stones) skipped horizontally by $P$. With this notation, we need to show that $(-1)^{b_{h}+w_{v}+r}=(-1)^{b_{h}+b_{v}+q}$, i.e., that $(-1)^{w_{v}+r}=(-1)^{b_{v}+q}$.

To prove this, we will show that $q-r=w_{v}+b_{v}$. Note that $q-r$ is the number of sources strictly between $s$ and $t$ which are not sources of any pseudopath in the pseudopath decomposition of $P$, that is, which are not in the set $\left\{j_{1}, \ldots, j_{r}\right\}$ from (6-2). Recall that the pseudopath decomposition of $P$ is a U-turn pseudopath decomposition; therefore, a source between $s$ and $t$ lies in $\left\{j_{1}, \ldots, j_{r}\right\}$ if and only if $P$ has a vertical edge ending at a + in this row. Otherwise $P$ skips a black or white stone in this row. This proves that $q-r=w_{v}+b_{v}$, and hence completes the proof of Theorem 6.6.

We have now shown that after an invertible transformation of the parameters, our network parametrization of $\mathscr{R}_{D}$ coincides with the corresponding MRparametrization of the projected Deodhar component $\mathscr{P}_{\boldsymbol{v}, \boldsymbol{w}}$. Combining this result with Proposition 4.3 yields Theorem 3.16.

Our proof yields the following statement.

Corollary 6.9. Let $D$ be the Go-diagram associated to the distinguished subexpression $\boldsymbol{v}$ of $\boldsymbol{w}$. Then $\mathscr{R}_{D}=\mathscr{P}_{\boldsymbol{v}, \boldsymbol{w}}$ as subsets of $\mathrm{Gr}_{k, n}$. Furthermore,

$$
\mathrm{Gr}_{k, n}=\bigsqcup_{D} \mathscr{R}_{D}
$$

where the union is over all Go-diagrams $D$ contained in a $k \times(n-k)$ rectangle.

Finally, we explain how our proof also yields Corollary 1.2.

Proof. We first note that the Marsh-Rietch parametrizations of Deodhar components restrict to parametrizations of cells in the totally nonnegative part of the complete flag variety (using Lusztig's definition of total positivity), if $\boldsymbol{v}$ is a positive distinguished subexpression of $\boldsymbol{w}$, and the parameters $p_{i}$ range over $\mathbb{R}_{>0}$ [Marsh and Rietsch 2004].

Our proof of Theorem 3.16 shows that if one takes a (particular) Marsh-Rietsch parametrization of a cell in the nonnegative part of the complete flag variety, then projects it to $\left(\mathrm{Gr}_{k, n}\right)_{\geq}$(using Lusztig's definition of total positivity), and then uses an invertible transformation of variables, one gets a network parametrization of a cell of $\left(\mathrm{Gr}_{k, n}\right)_{\geq 0}$ (using Postnikov's definition of total positivity). It follows that Lusztig's definition of $\left(\mathrm{Gr}_{k, n}\right)_{\geq 0}$ coincides with Postnikov's definition of $\left(\mathrm{Gr}_{k, n}\right)_{\geq 0}$, and moreover that the cell decompositions coincide. 


\section{A characterization of Deodhar components in terms of Plïcker coordinates}

In this section we characterize Deodhar components in the Grassmannian by a list of vanishing and nonvanishing Plücker coordinates. Our main result in this section is Theorem 7.8. The proof uses results from [Kodama and Williams 2013], which gave formulas for Plücker coordinates of Deodhar components.

\section{A. Plïcker coordinates of Deodhar components in terms of the MR parameters.}

Consider the Deodhar component $\mathscr{P}_{\boldsymbol{v}, \boldsymbol{w}} \subset \mathrm{Gr}_{k, n}$, where $\boldsymbol{w}$ is a reduced expression for $w \in W^{k}$ and $\boldsymbol{v} \prec \boldsymbol{w}$. In this section we will review some formulas from [Kodama and Williams 2013] for the Plücker coordinates of the elements of $\mathscr{P}_{\boldsymbol{v}, \boldsymbol{w}}$ in terms of the parameters which Marsh and Rietsch [2004] used to define $G_{v, w}$.

Theorem 7.1 [Kodama and Williams 2013, Lemma 5.1 and Theorem 5.2]. Let $\boldsymbol{w}=s_{i_{1}} \cdots s_{i_{m}}$ be a reduced expression for $w \in W^{k}$ and $\boldsymbol{v} \prec \boldsymbol{w}$ be a distinguished subexpression for $v$. Let $A=\pi_{k}(g) \in \mathscr{P}_{\boldsymbol{v}, \boldsymbol{w}}$ for any $g \in G_{\boldsymbol{v}, \boldsymbol{w}}$. Then the lexicographically minimal and maximal nonzero Plücker coordinates of $A$ are $\Delta_{I}$ and $\Delta_{I^{\prime}}$, where $I=w\{n, n-1, \ldots, n-k+1\}$ and $I^{\prime}=v\{n, n-1, \ldots, n-k+1\}$. If we write $g=g_{1} \cdots g_{m}$ as in Definition 4.1, then

$$
\Delta_{I}(A)=(-1)^{\left|J_{v}^{*}\right|} \prod_{i \in J_{v}^{+}} p_{i} \quad \text { and } \quad \Delta_{I^{\prime}}(A)=1 .
$$

Remark 7.2. If we write $I=\left\{i_{1}, \ldots, i_{k}\right\}$, then $I^{\prime}=v w^{-1}\left\{i_{1}, \ldots, i_{k}\right\}$.

Definition 7.3 [Kodama and Williams 2013, Definition 5.4]. Let $W=\mathfrak{S}_{n}$, let $\boldsymbol{w}=s_{i_{1}} \cdots s_{i_{m}}$ be a reduced expression for $w \in W^{k}$ and choose $\boldsymbol{v} \prec \boldsymbol{w}$. This determines a Go-diagram $D$ of shape $\lambda=\lambda_{w}$. Let $b$ be any box of $D$. Note that the set of all boxes of $D$ which are weakly southeast of $b$ forms a Young diagram $\lambda_{b}^{\text {in }}$; also the complement of $\lambda_{b}^{\text {in }}$ in $\lambda$ is a Young diagram which we call $\lambda_{b}^{\text {out }}$ (see Example 7.4 below). By looking at the restriction of $\boldsymbol{w}$ to the positions corresponding to boxes of $\lambda_{b}^{\text {in }}$, we obtain a reduced expression $\boldsymbol{w}_{b}^{\text {in }}$ for some permutation $w_{b}^{\text {in }}$, together with a distinguished subexpression $\boldsymbol{v}_{b}^{\text {in }}$ for some permutation $v_{b}^{\text {in }}$. Similarly, by using the positions corresponding to boxes of $\lambda_{b}^{\text {out }}$, we obtain $\boldsymbol{w}_{b}^{\text {out }}, w_{b}^{\text {out }}, \boldsymbol{v}_{b}^{\text {out }}$, and $v_{b}^{\text {out }}$. When the box $b$ is understood, we will often omit the subscript $b$.

For any box $b$, note that it is always possible to choose a reading order of $\lambda=\lambda_{w}$ which orders all the boxes of $\lambda^{\text {out }}$ after those of $\lambda^{\text {in }}$. We can then adjust $\boldsymbol{w}$ accordingly; this does not affect whether the corresponding expression $\boldsymbol{v}$ is distinguished. Having chosen such a reading order, we can then write $\boldsymbol{w}=\boldsymbol{w}^{\text {in }} \boldsymbol{w}^{\text {out }}$ and $\boldsymbol{v}=\boldsymbol{v}^{\text {in }} \boldsymbol{v}^{\text {out }}$. We then use $g^{\text {in }}$ and $g^{\text {out }}$ to denote the corresponding factors of $g \in G_{\boldsymbol{v}, \boldsymbol{w}}$. We define $J_{\boldsymbol{v}^{\text {out }}}^{+}$to be the subset of $J_{\boldsymbol{v}}^{+}$coming from the factors of $\boldsymbol{v}$ contained in $\boldsymbol{v}^{\text {out }}$. Similarly for $J_{\boldsymbol{v}^{\text {out }}}^{\circ}$ and $J_{\boldsymbol{v}^{\text {out }}}^{\bullet}$. 
Example 7.4. Let $W=\mathfrak{S}_{7}$ and $\boldsymbol{w}=s_{4} s_{5} s_{2} s_{3} s_{4} s_{6} s_{5} s_{1} s_{2} s_{3} s_{4}$ be a reduced expression for $w \in W^{3}$. Let $\boldsymbol{v}=s_{4} s_{5} 11 s_{4} 1 s_{5} s_{1} 11 s_{4}$ be a distinguished subexpression. So $w=(3,5,6,7,1,2,4)$ and $v=(2,1,3,4,6,5,7)$. We can represent this data by the poset $\lambda_{w}$ and the corresponding Go-diagram:

\begin{tabular}{|l|l|l|l|}
\hline$s_{4}$ & $s_{3}$ & $s_{2}$ & $s_{1}$ \\
\hline$s_{5}$ & $s_{4}$ & $s_{3}$ & $s_{2}$ \\
\hline$s_{6}$ & $s_{5}$ & $s_{4}$ & \multicolumn{1}{|c}{} \\
\cline { 1 - 2 }
\end{tabular}

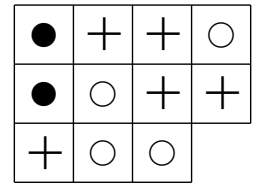

Let $b$ be the box of the Young diagram which is in the second row and the second column (counting from left to right). Then the diagram below shows: the boxes of $\lambda^{\text {in }}$ and $\lambda^{\text {out}}$; a reading order which puts the boxes of $\lambda^{\text {out }}$ after those of $\lambda^{\text {in }}$; and the corresponding labeled Go-diagram. Using this reading order, $\boldsymbol{w}^{\text {in }}=s_{4} s_{5} s_{2} s_{3} s_{4}$, $\boldsymbol{w}^{\text {out }}=s_{6} s_{5} s_{1} s_{2} s_{3} s_{4}, \boldsymbol{v}^{\text {in }}=s_{4} s_{5} 11 s_{4}$, and $\boldsymbol{v}^{\text {out }}=1 s_{5} s_{1} 11 s_{4}$.

\begin{tabular}{|l|l|l|l|}
\hline out & out & out & out \\
\hline out & in & in & in \\
\hline out & in & in & \multicolumn{1}{|c}{} \\
\cline { 1 - 2 }
\end{tabular}

\begin{tabular}{|l|l|l|l|}
\hline 11 & 10 & 9 & 8 \\
\hline 7 & 5 & 4 & 3 \\
\hline 6 & 2 & 1 & \multicolumn{1}{|c}{} \\
\cline { 1 - 2 } & &
\end{tabular}

\begin{tabular}{|c|c|c|c|}
\hline$-1 / m_{11}$ & $p_{10}$ & $p_{9}$ & 1 \\
\hline$-1 / m_{7}$ & 1 & $p_{4}$ & $p_{3}$ \\
\hline$p_{6}$ & 1 & 1 & \multicolumn{1}{|c}{} \\
\cline { 1 - 2 } & \multicolumn{2}{|c}{} &
\end{tabular}

Note that $J_{\boldsymbol{v}^{\text {out }}}^{\bullet}=\{7,11\}$ and $J_{\boldsymbol{v}^{\text {out }}}^{+}=\{6,9,10\}$. Then $g \in G_{\boldsymbol{v}, \boldsymbol{w}}$ has the form $g=g^{\text {in }} g^{\text {out }}=\left(\dot{s}_{4} \dot{s}_{5} y_{2}\left(p_{3}\right) y_{3}\left(p_{4}\right) \dot{s}_{4}\right)\left(y_{6}\left(p_{6}\right) x_{5}\left(m_{7}\right) \dot{s}_{5}^{-1} \dot{s}_{1} y_{2}\left(p_{9}\right) y_{3}\left(p_{10}\right) x_{4}\left(m_{11}\right) s_{4}^{-1}\right)$.

When we project the resulting $7 \times 7$ matrix to its first three columns, we get the matrix

$$
A=\left(\begin{array}{ccccccc}
-p_{9} p_{10} & -p_{3} p_{10} & -p_{10} & -m_{11} & 0 & -1 & 0 \\
0 & -p_{3} p_{4} & -p_{4} & -m_{7} & 1 & 0 & 0 \\
0 & 0 & 0 & p_{6} & 0 & 0 & 1
\end{array}\right)
$$

Theorem 7.5 [Kodama and Williams 2013, Theorem 5.6]. Let $\boldsymbol{w}=s_{i_{1}} \cdots s_{i_{m}}$ be a reduced expression for $w \in W^{k}$ and $\boldsymbol{v} \prec \boldsymbol{w}$, and let $D$ be the corresponding Godiagram. Choose any box $b$ of $D$, and let $v^{\text {in }}=v_{b}^{\text {in }}$ and $w^{\text {in }}=w_{b}^{\text {in }}$, and $v^{\text {out }}=v_{b}^{\text {out }}$ and $w^{\text {out }}=w_{b}^{\text {out }}$. Let $A=\pi_{k}(g)$ for any $g \in G_{\boldsymbol{v}, \boldsymbol{w}}$, and let $I=w\{n, n-1, \ldots, n-k+1\}$. If $b$ contains $a+$, define $I_{b}=v^{\text {in }}\left(w^{\text {in }}\right)^{-1} I \in\left(\begin{array}{c}{[n]} \\ k\end{array}\right)$. If $b$ contains a white or black stone, define $I_{b}=v^{\mathrm{in}} s_{b}\left(w^{\mathrm{in}}\right)^{-1} I \in\left(\begin{array}{c}{[n]} \\ k\end{array}\right)$. If we write $g=g_{1} \cdots g_{m}$ as in Definition 4.1, then:

(1) If $b$ contains $a+$, then $\Delta_{I_{b}}(A)=(-1)^{\left|J_{v^{\circ}}^{\text {out }}\right|} \prod_{i \in J_{v^{\text {out }}}^{+}} p_{i}$.

(2) If $b$ contains a white stone, then $\Delta_{I_{b}}(A)=0$.

(3) If b contains a black stone, then

$$
\Delta_{I_{b}}(A)=(-1)^{\left|J_{\boldsymbol{v}^{\text {out }}}\right|+1} m_{b} \prod_{i \in J_{\boldsymbol{v}^{\text {out }}}^{+}} p_{i}+\Delta_{I_{b}}\left(A_{b}\right)
$$


where $m_{b}$ is the parameter corresponding to $b$, and $A_{b}$ is the matrix $A$ with $m_{b}=0$.

Example 7.6. We continue Example 7.4. By Theorem 7.1, $I=w\{5,6,7\}=\{1,2,4\}$ and $I^{\prime}=v\{5,6,7\}=\{5,6,7\}$, and the lexicographically minimal and maximal nonzero Plücker coordinates for $A$ are $\Delta_{I}(A)=p_{3} p_{4} p_{6} p_{9} p_{10}$ and $\Delta_{I^{\prime}}(A)=1$; this can be verified for the matrix $A$ above.

We now verify Theorem 7.5 for the box $b$ labeled 5 in the reading order. Then $I_{b}=v^{\text {in }}\left(w^{\text {in }}\right)^{-1} I=\{1,4,6\}$. Theorem 7.5 says that $\Delta_{I_{b}}(A)=0$, since this box contains a white stone. The analogous computations for the boxes labeled 7, 6, 4, 3, 2,1 , respectively, yield $\Delta_{1,5,7}=-p_{9} p_{10}, \Delta_{1,2,7}=p_{3} p_{4} p_{9} p_{10}, \Delta_{1,4,5}=p_{6} p_{9} p_{10}$, $\Delta_{1,3,4}=p_{4} p_{6} p_{9} p_{10}, \Delta_{1,2,4}=p_{3} p_{4} p_{6} p_{9} p_{10}$, and $\Delta_{1,2,4}=p_{3} p_{4} p_{6} p_{9} p_{10}$. These can be checked for the matrix $A$ above.

Proposition 7.7 [Kodama and Williams 2013, Corollary 5.11]. For any box b, the rescaled Plücker coordinate

$$
\frac{\Delta_{I_{b}}(A)}{\prod_{i \in J_{v}^{+}} p_{i}}
$$

depends only on the parameters $p_{b^{\prime}}$ and $m_{b^{\prime}}$ which correspond to boxes $b^{\prime}$ weakly southeast of $b$ in the Go-diagram.

7B. The characterization of Deodhar components in terms of minors. Given a Go-diagram $D$ of shape $\lambda$, contained in a $k \times(n-k)$ rectangle, let $I=I(\lambda)$. It is not hard to check that if $D$ corresponds to the distinguished subexpression $\boldsymbol{v}$ of the reduced expression $\boldsymbol{w}$, then $I=w\{n, n-1, \ldots, n-k+1\}$.

Theorem 7.8. Let $D$ be a Go-diagram of shape $\lambda$ contained in a $k \times(n-k)$ rectangle. Let $A \in \mathrm{Gr}_{k, n}$. Then A lies in the Deodhar component $\mathscr{R}_{D}$ if and only if the following conditions are satisfied:

(1) $\Delta_{I_{b}}(A)=0$ for all boxes in $D$ containing a white stone.

(2) $\Delta_{I_{b}}(A) \neq 0$ for all boxes in $D$ containing $a+$.

(3) $\Delta_{I(\lambda)}(A) \neq 0$.

(4) $\Delta_{J}(A)=0$ for all $k$-subsets $J$ which are lexicographically smaller than $I(\lambda)$.

Proof. Suppose that $A$ lies in the Deodhar component $\mathscr{R}_{D}$. Then by Theorem 7.1, conditions (3) and (4) hold. And by Theorem 7.5, conditions (1) and (2) hold.

Now suppose that $A \in \mathrm{Gr}_{k, n}$, and conditions (1)-(4) hold. We want to show that $A \in \mathscr{R}_{D}$. Since the Deodhar components partition $\mathrm{Gr}_{k, n}$, it suffices to show that $A$ cannot lie in $\mathscr{R}_{D^{\prime}}$ for any other Go-diagram $D^{\prime}$. For the sake of contradiction, assume that $A \in \mathscr{R}_{D^{\prime}}$. Then by conditions (3) and (4), and Theorem 7.1, it follows that $D$ and $D^{\prime}$ must be Go-diagrams of the same shape. Therefore $D$ and $D^{\prime}$ correspond to distinguished subexpressions $\boldsymbol{v}$ and $\boldsymbol{v}^{\prime}$ of the same reduced expression $\boldsymbol{w}$. 
Choose a reading order for the boxes of the Go-diagrams $D$ and $D^{\prime}$, and let $b$ be the first box in that order where $D$ and $D^{\prime}$ differ. Then without loss of generality, in $D$ the box $b$ must contain a + , and in $D^{\prime}$ the box $b$ must contain a stone. (Because $\boldsymbol{v}$ and $\boldsymbol{v}^{\prime}$ are distinguished subexpressions of the same reduced word $\boldsymbol{w}$, which agree in the first $\ell$ factors and differ in the $(\ell+1)$-st factor, one of $\boldsymbol{v}$ and $\boldsymbol{v}^{\prime}$ must use the $(\ell+1)$-st simple generator $s_{b}$ and one must omit it.) In fact, it follows from the definition of distinguished subexpression and the fact that $D$ corresponds to a distinguished subexpression that the box $b$ in $D^{\prime}$ must contain a white stone, not a black one. (When building a distinguished subexpression from left to right, if choosing the next simple generator $s_{b}$ would decrease the length of the word so far, then one must choose $s_{b}$.)

Now note that the minor which Theorem 7.5 associates to box $b$ in $D$ is $\Delta_{I_{b}}$, where $I_{b}=v^{\text {in }}\left(w^{\text {in }}\right)^{-1}(I)$, and the minor which the theorem associates to box $b$ in $D^{\prime}$ is $\Delta_{I_{b}^{\prime}}$, where $I_{b}^{\prime}=v^{\prime \text { in }} s_{b}\left(w^{\text {in }}\right)^{-1}(I)$. But now note that $v^{\text {in }} s_{b}=v^{\text {in }}$. Therefore $I_{b}=I_{b}^{\prime}$. So then by Theorem 7.5, if $A \in \mathscr{R}_{D^{\prime}}$, then $\Delta_{I_{b}}(A)=0$, while by condition (2), $\Delta_{I_{b}}(A) \neq 0$. This is a contradiction.

Corollary 7.9. The Deodhar decomposition of the Grassmannian is a coarsening of the matroid stratification: in other words, each Deodhar component is a union of matroid strata.

Proof. Each matroid stratum is defined by specifying that certain Plücker coordinates are nonzero while the rest are zero. Therefore the corollary is an immediate consequence of Theorem 7.8.

There is also an oriented version of Corollary 7.9. To state this, we need a little preparation. First we define the oriented matroid stratification of the real Grassmannian to be the decomposition into strata based on which Plücker coordinates are 0 , positive, and negative. Next note that from Definition 4.1 and Proposition 4.3, it is immediate that if we are working over $\mathbb{K}=\mathbb{R}$, then the Deodhar component coming from a Go-diagram $D$ has $2^{r}$ connected components, where $r$ is the number of boxes in $D$ which contain a + . We have the following result.

Corollary 7.10. Consider the decomposition of the real Grassmannian into connected components of Deodhar components. This is a coarsening of the oriented matroid stratification: in other words, each connected component of a Deodhar component is a union of oriented matroid strata.

Proof. From Definition 4.1 and Proposition 4.3, we see that the connected components of a Deodhar component coming from $D$ are in bijection with the $2^{r}$ ways of choosing a sign (either positive or negative) for each of the $r$ parameters $p_{\ell}$ corresponding to the + boxes of $D$. By Theorems 7.1 and 7.5, such a choice of signs determines the sign pattern for the Plücker coordinates $\Delta_{I(\lambda)}$ and $\Delta_{I_{b}}$, 
where $b$ contains a + . Conversely, suppose we know the signs for those Plücker coordinates. Then we may algorithmically determine the signs of the $p_{\ell}$ 's: first we use Theorem 7.1 to determine the sign of the product of all of the $p_{\ell}$ 's; then we apply Theorem 7.5 to each box $b$ containing a + , reading the boxes in an order that proceeds from southeast to northwest. (For example, by reading the rows from bottom to top, and the boxes within each row from right to left.)

Remark 7.11. Theorem 7.8 implicitly gives an algorithm for determining the Deodhar component and corresponding network of a point of the Grassmannian, given by a matrix representative or by a list of its Plücker coordinates. The steps are as follows.

(1) Find the lexicographically minimal nonzero Plücker coordinate $\Delta_{I}$. Then the Go-diagram has shape $\lambda(I)$. Fix a reading order for this shape.

(2) We determine how to fill each box, working in the reading order, as follows. First check whether the box $b$ is forced to contain a black stone. If so, proceed to the next box. If not, look at $\Delta_{I_{b}}$. If this Plücker coordinate is zero, $b$ must contain a white stone, and if it is nonzero, $b$ must contain a + . Proceed to the next box. This process will completely determine the Go-diagram.

(3) Given the Go-diagram, we know what the underlying graph of the network must be. To determine the weights on horizontal edges, work through them in the reading order again. The Plücker coordinate $\Delta_{I_{b}}$ will only use edge weights $a_{b}$ (when $b$ contains a + ) or $c_{b}$ (when $b$ contains a black stone) and weights $a_{b^{\prime}}$ and $c_{b^{\prime}}$ corresponding to boxes $b^{\prime}$ which are earlier than $b$ in the reading order. Thus, we may use the Lindström-Gessel-Viennot lemma recursively to determine each weight $a_{b}$ or $c_{b}$.

\section{Acknowledgements}

We are grateful to Sara Billey for numerous helpful comments on the first version of this paper, as well as to an anonymous referee for useful comments. L. Williams is also grateful to Yuji Kodama for their joint work on soliton solutions of the KP equation, which provided motivation for this project.

\section{References}

[Björner and Brenti 2005] A. Björner and F. Brenti, Combinatorics of Coxeter groups, Graduate Texts in Mathematics 231, Springer, New York, 2005. MR 2006d:05001 Zbl 1110.05001

[Deodhar 1985] V. V. Deodhar, "On some geometric aspects of Bruhat orderings, I: A finer decomposition of Bruhat cells”, Invent. Math. 79:3 (1985), 499-511. MR 86f:20045 Zbl 0563.14023

[Deodhar 1987] V. V. Deodhar, "On some geometric aspects of Bruhat orderings, II: The parabolic analogue of Kazhdan-Lusztig polynomials”, J. Algebra 111:2 (1987), 483-506. MR 89a:20054 Zbl 0656.22007 
[Kazhdan and Lusztig 1979] D. Kazhdan and G. Lusztig, "Representations of Coxeter groups and Hecke algebras”, Invent. Math. 53:2 (1979), 165-184. MR 81j:20066 Zbl 0499.20035

[Kodama and Williams 2013] Y. Kodama and L. Williams, "The Deodhar decomposition of the Grassmannian and the regularity of KP solitons", Adv. Math. 244 (2013), 979-1032. MR 3077895

[Lusztig 1998] G. Lusztig, "Total positivity in partial flag manifolds", Represent. Theory 2 (1998), 70-78. MR 2000b:20060 Zbl 0895.14014

[Marsh and Rietsch 2004] R. J. Marsh and K. Rietsch, "Parametrizations of flag varieties", Represent. Theory 8 (2004), 212-242. MR 2005c:14061 Zbl 1053.14057

[Postnikov 2006] A. Postnikov, “Total positivity, Grassmannians, and networks”, preprint, 2006. arXiv math/0609764

[Proctor 1984] R. A. Proctor, "Bruhat lattices, plane partition generating functions, and minuscule representations”, European J. Combin. 5:4 (1984), 331-350. MR 86h:17007 Zbl 0562.05003

[Rietsch 1998] K. C. Rietsch, Total positivity and real flag varieties, thesis, Massachusetts Institute of Technology, 1998, Available at http://hdl.handle.net/1721.1/46139. MR 2716793

[Stembridge 1996] J. R. Stembridge, "On the fully commutative elements of Coxeter groups", J. Algebraic Combin. 5:4 (1996), 353-385. MR 97g:20046 Zbl 0864.20025

Communicated by Andrei Zelevinsky

Received 2012-10-19 Revised 2013-03-15 Accepted 2013-03-24

kellicar@umich.edu

williams@math.berkeley.edu
Department of Mathematics, University of California, Berkeley, 889 Evans Hall, Berkeley, CA 94720-3840, United States

Department of Mathematics, University of California, Berkeley, 913 Evans Hall, Berkeley, CA 94720-3840, United States 


\section{Algebra \& Number Theory}

msp.org/ant

\section{EDITORS}

MANAGING EDITOR

Bjorn Poonen

Massachusetts Institute of Technology

Cambridge, USA

\author{
EDITORIAL BOARD CHAIR \\ David Eisenbud \\ University of California \\ Berkeley, USA
}

\section{BOARD OF EDITORS}

Georgia Benkart

Dave Benson

Richard E. Borcherds

John H. Coates

J-L. Colliot-Thélène

Brian D. Conrad

Hélène Esnault

Hubert Flenner

Edward Frenkel

Andrew Granville

Joseph Gubeladze

Roger Heath-Brown

Ehud Hrushovski

Craig Huneke

Mikhail Kapranov

Yujiro Kawamata

János Kollár

Yuri Manin

Barry Mazur

Philippe Michel
University of Wisconsin, Madison, USA

University of Aberdeen, Scotland

University of California, Berkeley, USA

University of Cambridge, UK

CNRS, Université Paris-Sud, France

University of Michigan, USA

Freie Universität Berlin, Germany

Ruhr-Universität, Germany

University of California, Berkeley, USA

Université de Montréal, Canada

San Francisco State University, USA

Oxford University, UK

Hebrew University, Israel

University of Virginia, USA

Yale University, USA

University of Tokyo, Japan

Princeton University, USA

Northwestern University, USA

Harvard University, USA

École Polytechnique Fédérale de Lausanne
Susan Montgomery

Shigefumi Mori

Raman Parimala

Jonathan Pila

Victor Reiner

Karl Rubin

Peter Sarnak

Joseph H. Silverman

Michael Singer

Vasudevan Srinivas

J. Toby Stafford

Bernd Sturmfels

Richard Taylor

Ravi Vakil

Michel van den Bergh

Marie-France Vignéras

Kei-Ichi Watanabe

Efim Zelmanov

Shou-Wu Zhang
University of Southern California, USA

RIMS, Kyoto University, Japan

Emory University, USA

University of Oxford, UK

University of Minnesota, USA

University of California, Irvine, USA

Princeton University, USA

Brown University, USA

North Carolina State University, USA

Tata Inst. of Fund. Research, India

University of Michigan, USA

University of California, Berkeley, USA

Harvard University, USA

Stanford University, USA

Hasselt University, Belgium

Université Paris VII, France

Nihon University, Japan

University of California, San Diego, USA

Princeton University, USA

PRODUCTION

production@msp.org

Silvio Levy, Scientific Editor

See inside back cover or msp.org/ant for submission instructions.

The subscription price for 2013 is US $\$ 200 /$ year for the electronic version, and $\$ 350 /$ year $(+\$ 40$, if shipping outside the US) for print and electronic. Subscriptions, requests for back issues and changes of subscribers address should be sent to MSP.

Algebra \& Number Theory (ISSN 1944-7833 electronic, 1937-0652 printed) at Mathematical Sciences Publishers, 798 Evans Hall \#3840, c/o University of California, Berkeley, CA 94720-3840 is published continuously online. Periodical rate postage paid at Berkeley, CA 94704, and additional mailing offices.

ANT peer review and production are managed by EditFLOW ${ }^{\circledR}$ from Mathematical Sciences Publishers.

\section{PUBLISHED BY}

- mathematical sciences publishers

nonprofit scientific publishing

http://msp.org/

(C) 2013 Mathematical Sciences Publishers 


\section{Algebra \& Number Theory}

Volume $7 \quad$ No. $9 \quad 2013$

Multiplicities associated to graded families of ideals Steven Dale Cutkosky

Normal coverings of linear groups

JOHN R. BRITNELL and ATTILA MARÓTI

Modularity of the concave composition generating function

George E. ANDrews, Robert C. RhOAdes and SANDER P. ZWEgERS

ANDREW NILES

Regular permutation groups of order $m p$ and Hopf Galois structures

2203

ТIMOTHY KOHL

Further evidence for conjectures in block theory

2241

BENJAMIN SAMBALE

Network parametrizations for the Grassmannian

2275

Kelli TALASKa and LAUREN Williams

Chow quotients of toric varieties as moduli of stable log maps

2313

Qile Chen and Matthew Satriano

Vinberg's representations and arithmetic invariant theory

JACK A. THORNE 